\title{
THE MORDELL-WEIL GROUPS OF UNIRATIONAL QUASI-ELLIPTIC SURFACES IN CHARACTERISTIC 2
}

\author{
HIROYUKI ITO
}

(Received October 20, 1992, revised June 30, 1993)

\begin{abstract}
We continue to study the Mordell-Weil groups of unirational quasi-elliptic surfaces. We classify them in the case of rational quasi-elliptic surfaces in characteristic 2 and show how to construct them from the projective plane. In the classification, a key role is played by a theorem which guarantees that the relevant properties of unirational quasi-elliptic surfaces are determined explicitly by the equations of the surfaces as affine hypersurface.
\end{abstract}

1. Introduction. This is the first of a series of papers on the Mordell-Weil groups of unirational quasi-elliptic surfaces in characteristic 2. In our previous paper [5], we defined a group structure, which we call the Mordell-Weil group, on the set of $K$-rational points of a quasi-elliptic curve over the function field $K$ of a nonsingular complete curve, or equivalently, on the set of sections of a quasi-elliptic fibration over a nonsingular complete curve. In that paper, we proved that the Mordell-Weil group of a unirational quasi-elliptic surface is a torsion group and isomorphic to $(Z / p Z)^{\oplus r}$ with $r \geqslant 0$, and classified the degenerate fibers of a quasi-elliptic fibration, the torsion-rank $r$ of the Mordell-Weil group and the Néron-Severi group in the case where the characteristic of the ground field $k$ is 3 .

In the present paper, we continue to study the same question in the case of characteristic 2. Considerable differences and difficulties turn out to be involved, compared to the case of characteristic 3.

For example, the Weierstrass form of a quasi-elliptic curve in characteristic 2 is more complicated, and more cases have to be considered in the classification. In this paper, we treat the Weierstrass form of a quasi-elliptic curve over the rational function field in characteristic 2 and classify the degenerate fibers, the Mordell-Weil group and the Néron-Severi group of a rational quasi-elliptic surface in characteristic 2 . The first main theorem is Theorem 1.1 below, which generalizes a result in Miyanishi [7]. In Theorem 5.2 we classify degenerate fibers and the torsion-rank.

THEOREM 1.1. Let $k$ be an algebraically closed field of characteristic 2 and let

1991 Mathematics Subject Classification. Primary 14J27; Secondary 11G05, 14D99, 14J26.

Partly supported by the Grants-in-Aid for Scientific as well as Co-operative Research, The Ministry of Education, Science and Culture, Japan. 
$\mathscr{K}=k(x, y, t)$ be an algebraic function field of transcendence degree 2 generated by $x, y$, $t$ over $k$ such that $y^{2}=x^{3}+\varphi(t) x+\psi(t)$ with $\varphi(t), \psi(t) \in k[t]$ and either $\varphi(t) \notin k[t]^{2}$ or $\psi(t) \notin k[t]^{2}$. Define $m$ by

$$
m=\max \left\{\left[\frac{1}{4} \operatorname{deg} \varphi(t)\right],\left[\frac{1}{6} \operatorname{deg} \psi(t)\right]\right\},
$$

where $[z]$ signifies the greatest integer not exceeding $z$. Assume further that the following conditions hold:

(1) $\varphi(t)$ has no monomial terms of degree congruent to 0 modulo 4.

(2) $\psi(t)$ has no monomial terms of even degree.

(3) For every root $\alpha$ of $\varphi(t) \varphi^{\prime}(t)^{2}+\psi^{\prime}(t)^{2}=0$,

$$
\min \left\{v_{\alpha}(\varphi(t))-4, \quad v_{\alpha}(\psi(t))-6\right\}<0,
$$

where $v_{\alpha}$ is the $(t-\alpha)$-adic valuation of $k(t)$ so normalized that $v_{\alpha}(t-\alpha)=1$.

Then we have the following:

(a) If $m=0$, then $\mathscr{K}$ is rational over $k$. If $m \geqslant 1$, then $\mathscr{K}$ is not rational over $k$ and a minimal model $\hat{H}$ exists.

(b) If $m=1$, then $\hat{H}$ is a (supersingular) $K 3$ surface.

(c) If $m>1$, then $p_{a}(\hat{H})=p_{g}(\hat{H})=m, \operatorname{dim} H^{1}\left(\hat{H}, \mathcal{O}_{\hat{H}}\right)=0$, the r-genus $P_{r}(\hat{H})=$ $r(m-1)+1$ for every positive integer $r$, and the Kodaira dimension $\kappa(\hat{H})=1$, where $p_{a}$ is the arithmetic genus and $p_{g}$ is the geometric genus.

For the proof in Section 3, we use a method of double coverings as was used by Miyanishi [7] to obtain analogous results in characteristic 3 and partially in characteristic 2 , but we have to look into singularities of the ramification loci more carefully. Moreover, we exhibit the configuration of degenerate fibers of a rational quasi-elliptic surface and its sections, and show how to obtain it from $\boldsymbol{P}^{2}$ by blowing up nine points (see Section 5).

The author would like to thank Professors Masayoshi Miyanishi and De-Qi Zhang for stimulating discussions on this subject and Professor Igor V. Dolgachev for calling his attention to the book of Cossec-Dolgachev [4]. The author also extends his gratitude to the referee for precious advice on the improvement of this paper.

2. The Mordell-Weil group of a quasi-elliptic surface. Let $X$ be a nonsingular projective surface over an algebraically closed field $k$ of characteristic $p$. $X$ is said to be a quasi-elliptic surface if there exists a morphism $f: X \rightarrow C$ onto a nonsingular projective curve $C$ such that almost all fibers of $f$ are irreducible singular rational curves of arithmetic genus 1 . The morphism $f$ (or $X$ itself) is called a quasi-elliptic fibration. It is a well-known fact that such surfaces exist only if $p=2$ or 3 (cf. [10]).

In the present paper, we consider exclusively the case where $X$ is unirational, i.e., the case where $C \cong \boldsymbol{P}^{1}$ (cf. [7]), and assume that every quasi-elliptic surface has a section, 
denoted by $O$, which is a morphism from $C$ to $X$ such that $f \circ O=\mathrm{id}_{C}$.

For a quasi-elliptic surface $f: X \rightarrow C$, we use the following notation:

$X_{\eta}:$ the generic fiber of $f: X \rightarrow C$

$P_{\infty}$ : the unique singular point of $X_{\eta}$ (cf. [7])

$K=k(C)$ : the function field of $C$

$E:=X_{\eta}-P_{\infty}$ which is a smooth algebraic curve over $K$

$R=\left\{v \in C ; f^{-1}(v)\right.$ is reducible $\}$

$f^{-1}(v)=\Theta_{v, 0}+\sum_{i=1}^{m_{v}-1} \mu_{v, i} \Theta_{v, i}:$ the irreducible decomposition of $f^{-1}(v)$ for $v \in R$, where $\Theta_{v, i}\left(0 \leqslant i \leqslant m_{v}-1\right)$ are the irreducible components of $f^{-1}(v), m_{v}$ is the number of the irreducible components and $\Theta_{v, 0}$ is the unique component of $f^{-1}(v)$ meeting the zero section $(O)$.

$E(K)$ : the Mordell-Weil group, which is, by the definition, the set of all $K$-rational points of $E$, and endowed with a natural structure of the additive group (cf. $[5])$.

$\mathrm{NS}(X)$ : the Néron-Severi group of $X$

$T$ : the trivial lattice, which is, by definition, the subgroup of the Neron-Severi group generated by the zero section $(O)$ and all the irreducible components of the fibers.

For every $P \in E(K)$, we denote by $(P)$ the prime divisor of $X$ which is the image of $C$ by the morphism $C \rightarrow X$ induced by $P$, and we define a map $\varphi$ from $E(K)$ to $\mathrm{NS}(X)_{\boldsymbol{Q}}=\mathrm{NS}(X) \otimes_{\mathbf{Z}} Q$ by

$$
\varphi(P)=(P)-(O)-\left((P \cdot O)-\left(O^{2}\right)\right) F-\sum_{v \in R}\left(\Theta_{v, 1}, \ldots, \Theta_{v, m_{v}-1}\right) A_{v}^{-1}\left(\begin{array}{c}
\left(\Theta_{v, 1} \cdot P\right) \\
\vdots \\
\left(\Theta_{v, m_{v}-1} \cdot P\right)
\end{array}\right),
$$

where $(P \cdot O)$ is the abbreviation for $((P) \cdot(O))$ and $A_{v}$ is the negative definite matrix $\left(\left(\Theta_{v, i} \cdot \Theta_{v, j}\right)\right)_{i, j \geqslant 1}$ of size $\left(m_{v}-1\right)$. This $\varphi$ is a homomorphism and induces a natural isomorphism

$$
E(K) \simeq \mathrm{NS}(X) / T
$$

(cf. $[5, \S 2])$. We can define the height pairing $\langle$,$\rangle on E(K)$ via this isomorphism,

$$
\langle P, Q\rangle=-(\varphi(P) \cdot \varphi(Q))
$$

for $P, Q \in E(K)$, where the right hand side is induced by the intersection pairing on $\operatorname{NS}(X)_{\boldsymbol{Q}}$. The explicit form of this height pairing is

$$
\langle P, Q\rangle=\chi+(P \cdot O)+(Q \cdot O)-(P \cdot Q)-\sum_{v \in R} \operatorname{Contr}_{v}(P, Q),
$$

where 


$$
\operatorname{Contr}_{v}(P, Q)=\left\{\begin{aligned}
0 & \text { if } \quad P \text { or } Q \text { passes through } \Theta_{v, 0} \\
\left(-A_{v}^{-1}\right)_{i j} & \text { if } \quad\left(P \cdot \Theta_{v, i}\right)=\left(Q \cdot \Theta_{v, j}\right)=1 \text { with } i, j \geqslant 1,
\end{aligned}\right.
$$

and $\chi$ is the Euler-Poincaré characteristic of $X$. In particular,

$$
\langle P, P\rangle=2 \chi+2(P \cdot O)-\sum_{v \in R} \operatorname{Contr}_{v}(P),
$$

where

$$
\operatorname{Contr}_{v}(P)=\left\{\begin{aligned}
0 & \text { if } P \text { passes through } \Theta_{v, 0} \\
\left(-A_{v}^{-1}\right)_{i i} & \text { if }\left(P \cdot \Theta_{v, i}\right)=1 \text { with } i \geqslant 1,
\end{aligned}\right.
$$

(cf. [8]).

3. Proof of Theorem 1.1. Assume $p=2$. As in Miyanishi [7, (1.4)], $X$ is birational over $k$ to a hypersurface in the affine 3-space $A^{3}$ defined by

$$
y^{2}=x^{3}+\varphi(t) x+\psi(t)
$$

with $\varphi(t), \psi(t) \in k[t]$ such that either $\varphi(t) \notin k[t]^{2}$ or $\psi(t) \notin k[t]^{2}$. Let

$$
\Delta(t)=\varphi(t) \varphi^{\prime}(t)^{2}+\psi^{\prime}(t)^{2} \in k[t],
$$

where $\varphi^{\prime}(t)$ and $\psi^{\prime}(t)$ are the derivatives of $\varphi(t)$ and $\psi(t)$, respectively. By a birational transformation $\tau=1 / t, \xi=x / t^{2 m+2}$ and $\eta=y / t^{3 m+3}, X$ is also birational to the affine hypersurface

$$
\eta^{2}=\xi^{3}+\Phi(\tau) \xi+\Psi(\tau),
$$

where $\Phi(\tau)=\tau^{4 m+4} \varphi(1 / \tau), \Psi(\tau)=\tau^{6 m+6} \psi(1 / \tau)$ and $m=\max \{[\operatorname{deg} \varphi(t) / 4],[\operatorname{deg} \psi(t) / 6]\}$. We set $\Delta_{\infty}(\tau)=\Phi(\tau) \Phi^{\prime}(\tau)^{2}+\Psi^{\prime}(\tau)^{2}$. By a straightforward computation, we have

$$
\Delta_{\infty}(\tau)=\tau^{12 m+8} \Delta\left(\frac{1}{\tau}\right)
$$

We call $\Delta(t)$ or $\Delta_{\infty}(\tau)$ the discriminant of $X$.

LEMMA 3.1. By a suitable choice of coordinates $(x, y, t)$, we may assume that the following conditions are satisfied:

(1) $\varphi(t)$ has no monomial terms of degree congruent to 0 modulo 4.

(2) $\psi(t)$ has no monomial terms of even degree.

(3) $\min \left\{v_{\alpha}(\varphi(t))-4, v_{\alpha}(\psi(t))-6\right\}<0$ for all roots $\alpha$ of $\Delta(t)=0$.

Proof. If the condition (3) is not satisfied for some root $\alpha$ of $\Delta(t)=0$, divide both sides of (3.1) by $(t+\alpha)^{6}$ and replace the coordinates $(x, y, t)$ by $\left(x /(t+\alpha)^{2}, y /(t+\alpha)^{3}, t\right)$. After a succession of operations of this kind, we may assume that the condition (3) is fulfilled. Suppose $\varphi(t)$ is written in the form 


$$
\varphi(t)=\varphi_{0}(t)+\varphi_{1}(t)^{4},
$$

with $\varphi_{0}(t), \varphi_{1}(t) \in k[t]$ such that $\varphi_{0}(t)$ satisfies the condition (1).

After a transformation $(x, y, t) \mapsto\left(x+\varphi_{1}(t)^{2}, y+\varphi_{1}(t) x+\varphi_{1}(t)^{3}, t\right)$ we have

$$
y^{2}=x^{3}+\varphi_{0}(t) x+\varphi_{0}(t) \varphi_{1}(t)^{2}+\psi(t)
$$

which satisfies the condition (3). As for the condition (2), the monomial terms of even degree in $\psi(t)$ can be absorbed by $y$-term.

REMARK 1. The above operations to get the conditions (1) and (2) fulfilled do not change the discriminant $\Delta(t)$.

Proof of Theorem 1.1 consists of the subsequent Lemmas 3.2-3.8. Let $\mathscr{K}$ be the algebraic function field as in Theorem 1.1. We assume that $\varphi(t)$ and $\psi(t)$ in the equation (3.1) satisfy the additional conditions in Lemma 3.1. A rough idea of our proof is, first of all, to construct a nonsingular model $H$ of $\mathscr{K}$ as a double covering of a rational surface obtained from $\boldsymbol{P}^{1} \times \boldsymbol{P}^{1}$ by blowing-up, which turns out to be a quasi-elliptic surface with a section, and secondly to contract all $(-1)$-curves contained in the fibers of the quasi-elliptic fibration to obtain a relatively minimal model $\hat{H}$ of $H$. Then, by virtue of Miyanishi [7], it suffices to show that $\chi\left(\mathcal{O}_{\hat{H}}\right)=m+1$ with the notation as in the statement of the theorem (cf. [7, Lemmas 1.5 and 1.6]).

LEMMA 3.2. After a change of coordinates $x$ and $y$, we can rewrite the equation (3.1) in the form

$$
y^{2}=x^{3}+\alpha(t) x^{2}+\beta(t) x+\gamma(t),
$$

where $\alpha(t), \beta(t), \gamma(t)$ are polynomials in $k[t]$ having no monomial terms of even degree and the integer $m$ defined in Theorem 1.1 is given as

$$
m=\max \left\{\left[\frac{1}{2} \operatorname{deg} \alpha(t)\right],\left[\frac{1}{4} \operatorname{deg} \beta(t)\right],\left[\frac{1}{6} \operatorname{deg} \gamma(t)\right]\right\} .
$$

Proof. Write $\varphi(t)=\alpha(t)^{2}+\beta(t)$ and $\psi(t)=\gamma(t)$ with $\alpha$ and $\beta$ having no monomial terms of even degree. Then the coordinate change $(x, y) \mapsto\left(x+\alpha, y+(\alpha \beta)^{1 / 2}\right)$ will give the above form with $\psi(t)=\gamma(t)$. It is easy to find that the integer $m$ in Theorem 1.1 is given as above.

Put $A(x, t)=x^{3}+\alpha(t) x^{2}+\beta(t) x+\gamma(t)$ as a polynomial in $k[x, t]$. Consider a hypersurface $y^{2}=A(x, t)$ in the projective 3 -space $\boldsymbol{P}_{k}^{3}$ with the affine $(x, y, t)$ space embedded in $\boldsymbol{P}_{k}^{3}$ as $(x, y, t) \mapsto[x, y, t, 1]$, which is birational to a double covering of $F_{0}:=\boldsymbol{P}_{k}^{1} \times \boldsymbol{P}_{k}^{1}$. Let $\rho_{0}: H_{0} \rightarrow F_{0}$ be the normalization of $F_{0}$ in $\mathscr{K}$. The equation $A(x, t)=0$ defines a closed curve $C$ on $F_{0}$.

Now we introduce the following notation: Consider the $\boldsymbol{P}^{1}$-fibration $\mathscr{F}:=\left\{l_{\alpha} ; l_{\alpha}\right.$ is defined by $t=\alpha\}$ on $F_{0}$ defined by the second projection $p_{2}: F_{0} \rightarrow \boldsymbol{P}_{k}^{1}$. We denote by $l_{\infty}$ 
the fiber $t=\infty$ and by $S_{\infty}$ the cross-section $x=\infty$. Let $\bar{\sigma}: \bar{F} \rightarrow F_{0}$ be the shortest succession of blowing-up of $F_{0}$ at the singular points of $C$ and their infinitely near singular points such that the proper transform $\bar{C}=\bar{\sigma}^{\prime}(C)$ of $C$ on $\bar{F}$ becomes nonsingular. Let $\bar{S}_{\infty}=\bar{\sigma}^{\prime}\left(S_{\infty}\right)$ and $\bar{l}_{\infty}:=\bar{\sigma}^{\prime}\left(l_{\infty}\right)$ be the proper transforms under $\bar{\sigma}$ of $S_{\infty}$ and $l_{\infty}$. We denote by $a, b, c$ the degrees of $\alpha(t), \beta(t), \gamma(t)$, respectively.

We shall describe the configuration of $C$ on $F_{0}$ and $\bar{\sigma}^{-1}\left(l_{\infty} \cup C \cup S_{\infty}\right)$ on $\bar{F}$.

LEMMA 3.3. (0) We distinguish the following four cases by means of the degrees of $\alpha(t), \beta(t)$ and $\gamma(t)$.

(I) $a=b \geqslant c$; (II) $b>a, b \geqslant c$; (III) $c>a, c>b$; (IV) $a>b, a \geqslant c$.

The configuration of $l_{\infty} \cup C \cup S_{\infty}$ on $F_{0}$ for each case is shown in Figure 1.

(1) In the case (I), C has no singular points on $l_{\infty}$ and $S_{\infty}$ and $\bar{\sigma}$ is the identity morphism.

(2) In the case (II), we have the following three cases:

(II-1) $a / 2 \leqslant b / 4$ and $b \equiv 1(\bmod 4) ;$ then $b=4 m+1, a \leqslant 2 m$ and $c<b$.

(II-2) $a / 2 \leqslant b / 4$ and $b \equiv 3(\bmod 4)$; then $b=4 m+3, a \leqslant 2 m+1$ and $c<b$.

(II-3) $a / 2>b / 4$.

The configurations of $\bar{\sigma}^{-1}\left(l_{\infty} \cup C \cup S_{\infty}\right)$ of these cases are given in Figure 2.

(3) In the case (III), we have the following three cases:

(III-1) $\max \{a / 2, b / 4\} \leqslant c / 6$.

(III-2) $\max \{a / 2, b / 4\}>c / 6$ and $a / 2 \leqslant b / 4$.

(III-3) $\max \{a / 2, b / 4\}>c / 6$ and $a / 2>b / 4$.

In the case (III-1), we have $m=[c / 6]$. Hence $c=6 m+1,6 m+3$ or $6 m+5$. According to the values of $C$, the case (III-1) is divided into three subcases.

(III-1-1) $c=6 m+1, a \leqslant 2 m$ and $b \leqslant 4 m$.

(III-1-2) $c=6 m+3, a \leqslant 2 m+1$ and $b \leqslant 4 m+1$.

(III-1-3) $c=6 m+5, a \leqslant 2 m+1$ and $b \leqslant 4 m+3$.

The configurations are given in Figure 3.

In the case (III-2), we have $m=[b / 4]$. Hence $b=4 m+1$ or $4 m+3$. According to the values of $b$, the case (III-2) is divided into two subcases.

(III-2-1) $\quad b=4 m+1, a \leqslant 2 m$ and $c \leqslant 6 m+1$.

(III-2-2) $b=4 m+3, a \leqslant 2 m+1$ and $c \leqslant 6 m+3$.

The configurations are given in Figure 3.

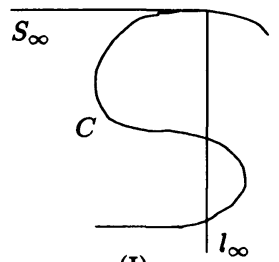

(I)

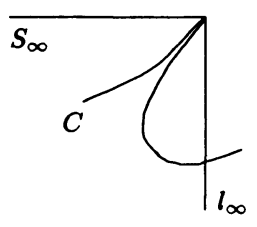

(II)

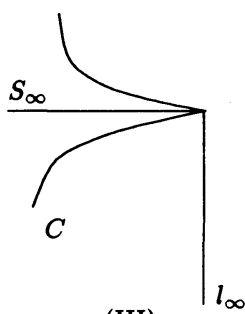

(III)

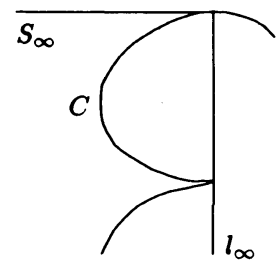

(IV)

Figure 1. 
Case (II-1)
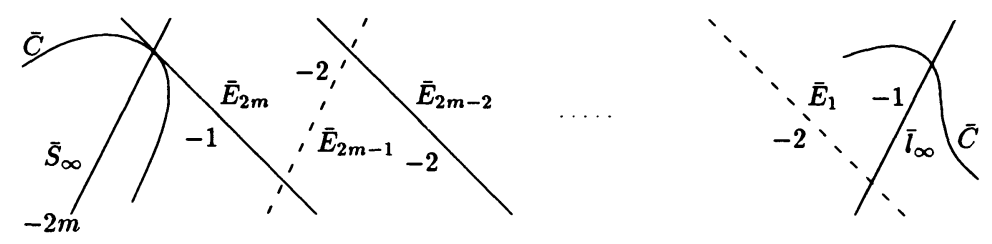

$\left(\bar{C} \cdot \bar{E}_{2 m}\right)=2$.

Case (II-2)
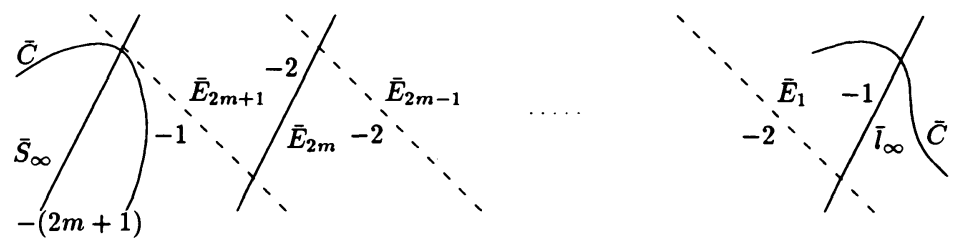

$\left(\bar{C} \cdot \bar{E}_{2 m+1}\right)=2$.

Case (II-3)
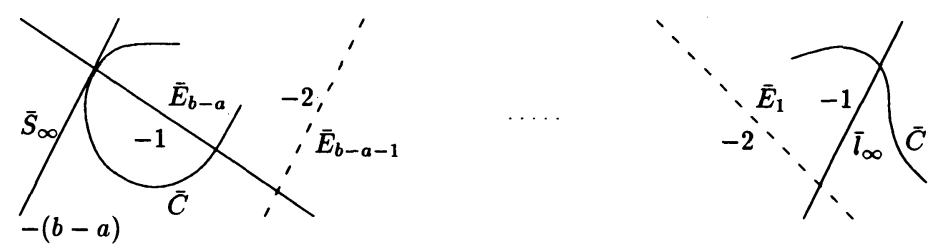

$$
\left(\bar{C} \cdot \bar{S}_{\infty}\right)=2 a-b \text { and }\left(\bar{C} \cdot \bar{E}_{b-a}\right)=2 .
$$

FIGURE 2.

In the case (III-3), we have $m=[a / 2]$. Hence $a=2 m+1$. In this case, we have three subcases.

$$
\begin{array}{ll}
\text { (III-3-1) } & (c-a) / 2=c-b . \\
\text { (III-3-2) } & (c-a) / 2<c-b . \\
\text { (III-3-3) } & (c-a) / 2>c-b .
\end{array}
$$

The configurations are given in Figure 3.

(4) In the case (IV), the configuration of $\bar{\sigma}^{-1}\left(l_{\infty} \cup C \cup S_{\infty}\right)$ is given in Figure 4.

Proof. (0) It is easy to see that the cases (I)-(IV) exhaust all possible cases. In each of the four cases, the configuration of $l_{\infty} \cup C \cup S_{\infty}$ is elucidated by locating the singular points of $C$ and writing a local equation of $C$ which will be treated below in each case.

(1) Write 
228

H. ITO

Case (III-1-1)
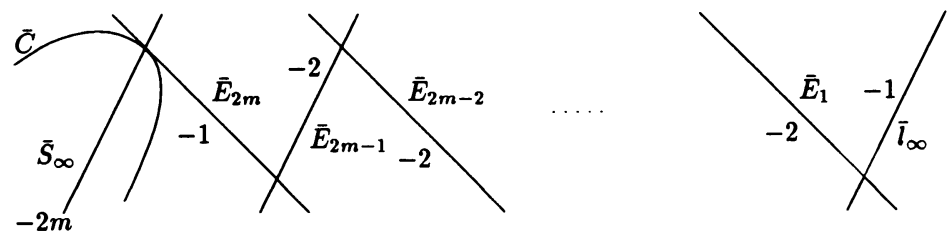

$\left(\bar{C} \cdot \bar{E}_{2 m}\right)=3$.

Case (III-1-2)
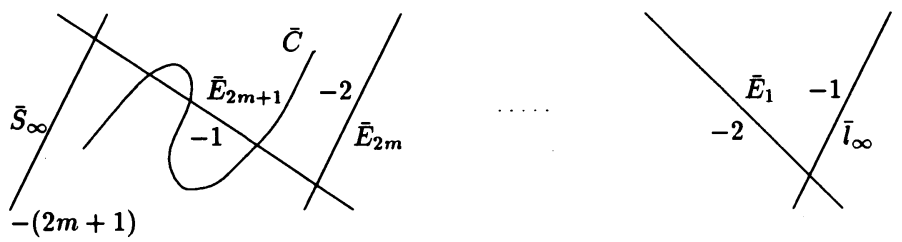

Case (III-1-3)
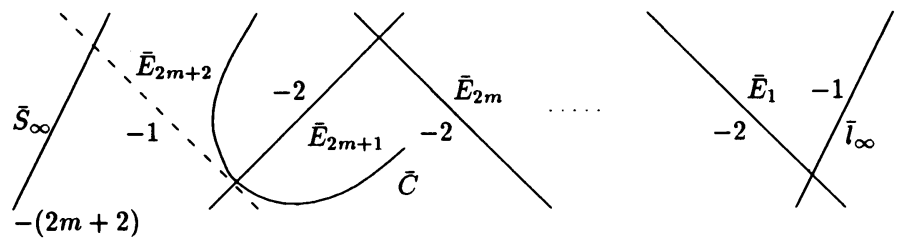

$\left(\bar{C} \cdot \bar{E}_{2 m+2}\right)=2$.

Case (III-2-1)
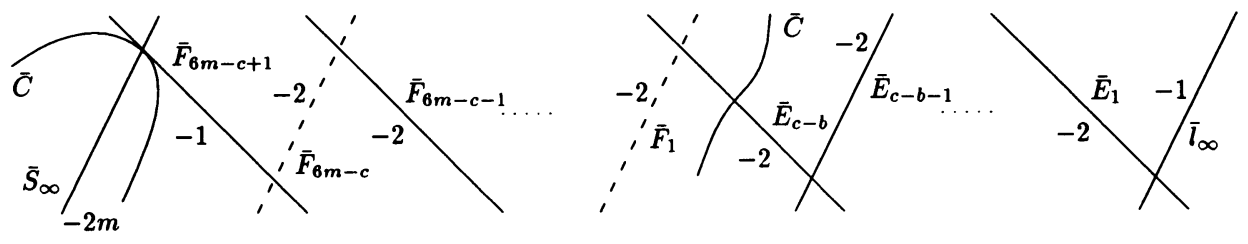

$\left(\bar{C} \cdot \bar{F}_{6 m-c+1}\right)=2$.

Case (III-2-2)
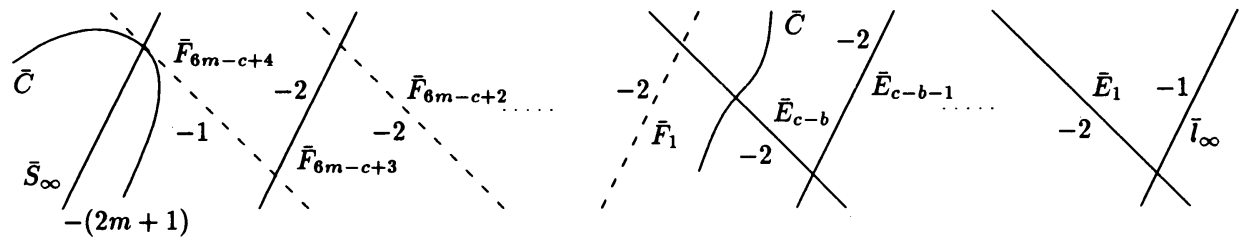

$\left(\bar{C} \cdot \bar{F}_{6 m-c+4}\right)=2$. 
Case (III-3-1)
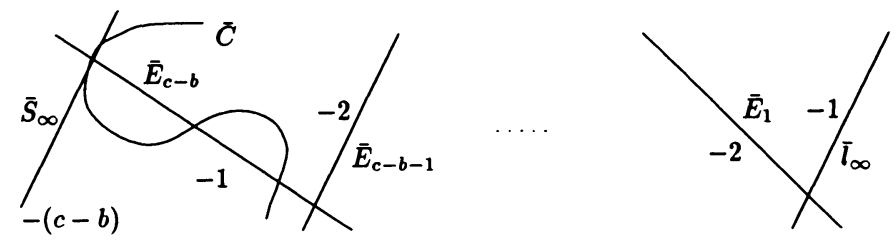

$$
\left(\bar{C} \cdot \bar{S}_{\infty}\right)=3 b-2 c .
$$

Case (III-3-2)
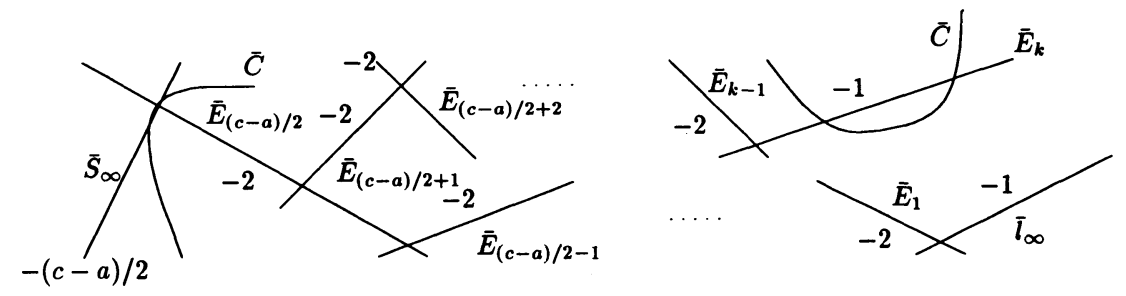

$\left(\bar{C} \cdot \bar{S}_{\infty}\right)=(3 a-c) / 2$ and $k$ is some positive integer.

\section{Case (III-3-3)}
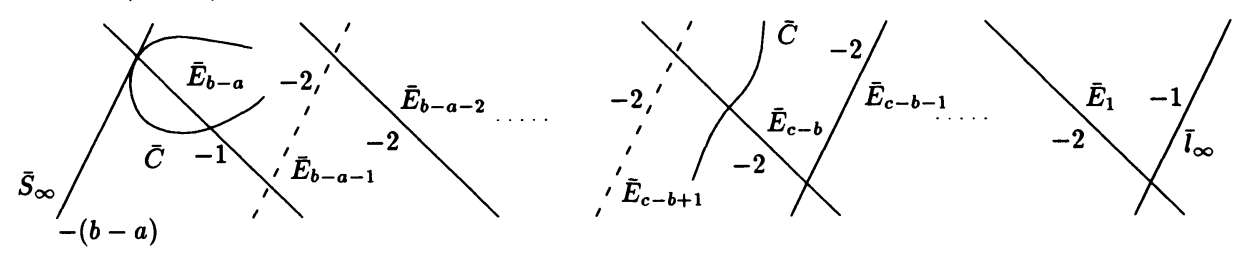

$\left(\bar{C} \cdot \bar{S}_{\infty}\right)=2 a-b$.

FIGURE 3.
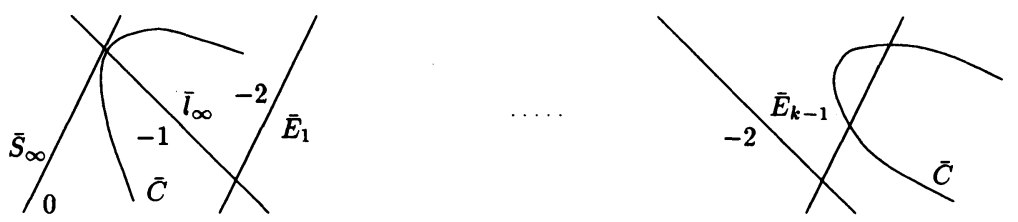

$\left(\bar{C} \cdot \bar{S}_{\infty}\right)=a$ and $k$ is some positive integer.

Figure 4.

$$
\begin{aligned}
& \alpha(t)=c_{0} t^{a}+c_{1} t^{a-1}+\cdots+c_{a} \\
& \beta(t)=d_{0} t^{b}+d_{1} t^{b-1}+\cdots+d_{b} \\
& \gamma(t)=e_{0} t^{c}+e_{1} t^{c-1}+\cdots+e_{c} .
\end{aligned}
$$


Then $\xi^{3} \tau^{a} A(1 / \xi, 1 / \tau)$ is written as

$$
\begin{aligned}
\tau^{a}+\left(c_{0}+c_{1} \tau+\cdots\right. & \left.+c_{a} \tau^{a}\right) \xi+\left(d_{0}+d_{1} \tau+\cdots+d_{b} \tau^{b}\right) \xi^{2} \\
& +\left(e_{0}+e_{1} \tau+\cdots+e_{c} \tau^{c}\right) \tau^{a-c} \xi^{3}=0,
\end{aligned}
$$

where $\xi=1 / x$ and $\tau=1 / t$, and we may assume $c_{0} d_{0} e_{0} \neq 0$. This is the equation for $C$ near the point $P_{\infty}: \xi=0$ and $\tau=0$, and it can be formally (analytically) written as $\xi+\tau^{a}=0$. Thus, $C$ is smooth at the points $P_{\infty}:=S_{\infty} \cap l_{\infty}$ and meets $S_{\infty}$ with order of contact $a$ and intersects $l_{\infty}$ transversally at three distinct points. Thence we obtain the above description for $C$ at $P_{\infty}$.

(2) In this case, $C$ has a singular point at $P_{\infty}:=S_{\infty} \cap l_{\infty}$, and $C$ intersects transversally $l_{\infty}$ at the point $(x, \tau)=(0,0)$, whre $\tau=1 / t$ (see Figure 1 ). Indeed, $C$ is formally written at $P_{\infty}$ as

$$
\tau^{b}+\left(c_{0}+c_{1} \tau+\cdots+c_{a} \tau^{a}\right) \tau^{b-a} \xi+\xi^{2}=0,
$$

where $l_{\infty}$ and $S_{\infty}$ are defined respectively by $\tau=0$ and $\xi=0$.

By blowing-up of $P_{\infty}$ and $(l-1)$ infinitely near points lying on $S_{\infty}$ we obtain coordinates $\xi_{1}, \ldots, \xi_{l}$ such that

$$
\xi=\tau \xi_{1}, \xi_{1}=\tau \xi_{2}, \ldots, \xi_{l-1}=\tau \xi_{l},
$$

and that the proper transform $\bar{C}$ of $C$ is given by

$$
\tau^{b-2 l}+\left(c_{0}+c_{1} \tau+\cdots+c_{a} \tau^{a}\right) \tau^{b-a-l} \xi_{l}+\xi_{l}^{2}=0
$$

provided $b \geqslant 2 l$ and $b \geqslant a+l$. To describe the configuration of $\bar{\sigma}^{-1}\left(l_{\infty} \cup C \cup S_{\infty}\right)$ on $\bar{F}$ we distinguish three subcases.

Consider first the case $b / 4 \geqslant a / 2$. Since $c \leqslant b$, we have $m=[b / 4]$ by Lemma 3.2. Noting that $b \not \equiv 0(\bmod 2)$ by hypothesis, we have either $b=4 m+1$ or $b=4 m+3$. If $b=4 m+1$ then $a \leqslant 2 m$ and we can take $l=2 m$. Then $\bar{C}$ is also smooth at the point $\left(\xi_{2 m}=0, \tau=0\right)$. This is the subcase (II-1) above. If $b=4 m+3$ then $a \leqslant 2 m+1$ and we can take $l=2 m+1$. Then $\bar{C}$ is smooth at the points $\left(\xi_{2 m+1}=0, \tau=0\right)$. This is the subcase (II-2) above. Consider next the case $b / 4<a / 2$. Then $m=[a / 2]$ and $a=2 m+1$. So, $b \leqslant 4 m+1$. In this case, we can take $l=b-a$, and $\bar{C}$ is written at the points $\left(\xi_{l}=0\right.$, $\tau=0)$ as

$$
\tau^{2 a-b}+\left(c_{0}+c_{1} \tau+\cdots+c_{a} \tau^{a}\right) \xi_{l}+\xi_{l}^{2}=0 .
$$

Since $c_{0} \neq 0, \bar{C}$ meets the curve $\bar{E}_{l}$ as two points $\xi_{l}=0$ and $\xi_{l}=c_{0}$. This is the subcase (II-3) above.

(3) The formal equation for $C$ at $P_{\infty}$ is given by

$$
\tau^{c}+\left(c_{0}+c_{1} \tau+\cdots+c_{a} \tau^{a}\right) \tau^{c-a} \xi+\left(d_{0}+d_{1} \tau+\cdots+d_{b} \tau^{b}\right) \tau^{c-b} \xi^{2}+\xi^{3}=0 .
$$

Thus we have $\left(C \cdot l_{\infty}\right)=3$ and $\left(C \cdot S_{\infty}\right)=c$ (see Figure 1).

As in Case II, the blowing-up of $P_{\infty}$ and $(r-1)$ infinitely near points of $P_{\infty}$ lying 
on $S_{\infty}$ allows us to introduce $\xi_{1}, \ldots, \xi_{r}$ such that

$$
\xi=\tau \xi_{1}, \xi_{1}=\tau \xi_{2}, \ldots, \xi_{r-1}=\tau \xi_{r}
$$

and that proper transform $\hat{C}$ of $C$ is defined by

$$
\tau^{c-3 r}+\left(c_{0}+c_{1} \tau+\cdots+c_{a} \tau^{a}\right) \tau^{c-a-2 r} \xi_{r}+\left(d_{0}+d_{1} \tau+\cdots+d_{b} \tau^{b}\right) \tau^{c-b-r} \xi_{r}^{2}+\xi_{r}^{3}=0
$$

provided $c \geqslant 3 r, c \geqslant a+2 r$ and $c \geqslant b+r$.

We further distinguish three cases.

Consider the case (III-1), where $m=[c / 6]$ by Lemma 3.2. Then we can take $r=2 m$, $r=2 m+1$ and $r=2 m+1$ according as $c=6 m+1, c=6 m+3$ and $c=6 m+5$. In the case $c=6 m+5$, i.e., the case (III-1-3), the equation for $\hat{C}$ is equivalent to

$$
\tau^{2}+\left(c_{0}+c_{1} \tau+\cdots+c_{a} \tau^{a}\right) \tau^{\geqslant 2} \xi_{r}+\left(d_{0}+d_{1} \tau+\cdots+d_{b} \tau^{b}\right) \tau^{\geqslant 1} \xi_{r}^{2}+\xi_{r}^{3}=0,
$$

where $\tau^{\geqslant 2}$ (or $\tau^{\geqslant 1}$ ) stands for a monic monomial in $\tau$ of degree $\geqslant 2$ (or $\geqslant 1$ ). So, we have to blow up the point $\left(\xi_{r}=0, \tau=0\right)$ to desingularize $\hat{C}$. The other cases can be handled similarly.

Consider the case (III-2), where $m=[b / 4]$. Then, for $r=c-b$, we have

$$
\tau^{3 b-2 c}+\left(c_{0}+c_{1} \tau+\cdots+c_{a} \tau^{a}\right) \tau^{2 b-a-c} \xi_{r}+\left(d_{0}+d_{1} \tau+\cdots+d_{b} \tau^{b}\right) \xi_{r}^{2}+\xi_{r}^{3}=0,
$$

where $3 b-2 c>0$ and $2 b-a-c>0$ under the hypothesis. So, $\hat{C}$ meets the last exceptional curve $E_{c-b}$ at two points $\left(\xi_{r}=0, \tau=0\right)$ and $\left(\xi_{r}=d_{0}, \tau=0\right)$, where the latter point is a smooth point of $\hat{C}$. Near the point $\left(\xi_{r}=0, \tau=0\right)$, the equation for $\hat{C}$ is formally equivalent to

$$
\tau^{3 b-2 c}+\left(c_{0}+c_{1} \tau+\cdots+c_{a} \tau^{a}\right) \tau^{2 b-a-c \zeta}+\zeta^{2}=0 .
$$

Introduce $\zeta_{1}, \ldots, \zeta_{s}$ by

$$
\zeta=\tau \zeta_{1}, \ldots, \zeta_{s-1}=\tau \zeta_{s}
$$

Then the equation becomes

$$
\tau^{3 b-2 c-2 s}+\left(c_{0}+c_{1} \tau+\cdots+c_{a} \tau^{a}\right) \tau^{2 b-a-c-s} \zeta_{s}+\zeta_{s}^{2}=0,
$$

provided $3 b-2 c-2 s \geqslant 0$ and $2 b-a-c-s \geqslant 0$. If $b=4 m+1$, i.e., the case (III-2-1), put $l=3 m-(c-1) / 2$ and $s=2 l$. Then

$$
3 b-2 c-2 s=1 \text { and } 2 b-a-c-s=2 m+1-a \geqslant 1 .
$$

So, the proper transform $\bar{C}$ of $\hat{C}$ after these $s$ blowing-up is smooth. If $b=4 m+3$, i.e., the case (III-2-2), put $l=3 m-(c-3) / 2$ and $s=2 l+1$. Then

$$
3 b-2 c-2 s=1 \text { and } 2 b-a-c-s=2 m+2-a \geqslant 1 .
$$

Hence $\bar{C}$ is also desingularized.

Consider the case (III-3), where $m=[a / 2]$. Looking at the equation 


$$
\tau^{c-3 r}+\left(c_{0}+c_{1} \tau+\cdots+c_{a} \tau^{a}\right) \tau^{c-a-2 r} \xi_{r}+\left(d_{0}+d_{1} \tau+\cdots+d_{b} \tau^{b}\right) \tau^{c-b-r} \xi_{r}^{2}+\xi_{r}^{3}=0
$$

for $\hat{C}$, we can now compare the $\tau$-degrees of the second and third terms and distinguish three cases:

(III-3-1) $c-a-2 r=0$ and $c-b-r=0$,

(III-3-2) $c-a-2 r=0$ and $c-b-r>0$,

(III-3-3) $c-a-2 r>0$ and $c-b-r=0$.

The desingularization process for $C$ at $P_{\infty}$ is more or less similar to those treated above. We only note that we need more attention in the case (III-3-2).

(4) This case can be treated in a similar fashion. Note that $C$ meets $l_{\infty}$ at two points $(\xi=0, \tau=0)$ and $(x=0, \tau=0)$ when $a>c,\left(x=e_{0} / c_{0}, \tau=0\right)$ when $a=c$, using the same notation (3.3), (3.4) in the case I, where the latter point is a singular point (Figure 1). The desingularization is as illustrated in Figure 4.

We may write $\left(\bar{\sigma}^{*} A\right)$ uniquely in the form $\left(\bar{\sigma}^{*} A\right)=\bar{B}-2 \bar{Z}$, where $\bar{B}$ is a divisor whose coefficient at each prime divisor is 0 or 1 and where $\bar{Z}$ is some divisor. Let $\bar{D}$ be an effective divisor whose support is contained in the union $\mathscr{E}$ of the exceptional curves which arise from the blowing-up at the singular points and their infinitely near points of $C$ in the affine part $A_{k}^{2}=F_{0}-S_{\infty} \cup l_{\infty}$. Let $\bar{D}_{1}$ and $\bar{D}_{2}$ be the divisors determined uniquely by the conditions that $\bar{D}_{1}$ is an effective divisor whose coefficient at each prime divisor is 0 or $1, \bar{D}_{2} \geqslant 0, \bar{D}_{1}+2 \bar{D}_{2}=\bar{D}$ and $\operatorname{Supp}\left(\bar{D}_{1} \cup \bar{D}_{2}\right) \subset \mathscr{E}$. We note that in the previous configuration of $\bar{\sigma}^{-1}\left(l_{\infty} \cup C \cup S_{\infty}\right)$, an irreducible component is drawn by an unbroken (resp. broken) line if it is contained in $\operatorname{Supp} \bar{B}$ (resp. not contained in $\operatorname{Supp} \bar{B}$ ). We consider four cases separately.

Case I. $a=b \geqslant c$

$$
\begin{aligned}
& \bar{B}=\bar{C}+\bar{l}_{\infty}+\bar{S}_{\infty}+\bar{D}_{1} \\
& \bar{Z}=2 \bar{S}_{\infty}+(m+1) \bar{l}_{\infty}-\bar{D}_{2} .
\end{aligned}
$$

Case II. $b>a, b \geqslant c$

(II-1)

$$
\begin{aligned}
\bar{B}= & \bar{C}+\bar{l}_{\infty}+\bar{S}_{\infty}+\left(\bar{E}_{2}+\bar{E}_{4}+\cdots+\bar{E}_{2 m}\right)+\bar{D}_{1} \\
\bar{Z}= & 2 \bar{S}_{\infty}+(2 m+1)\left(\bar{l}_{\infty}+\bar{E}_{1}\right)+(2 m+2)\left(\bar{E}_{2}+\bar{E}_{3}\right)+\cdots \\
& +3 m\left(\bar{E}_{2 m-2}+\bar{E}_{2 m-1}\right)+(3 m+1) \bar{E}_{2 m}-\bar{D}_{2} .
\end{aligned}
$$

$$
\begin{aligned}
\bar{B}= & \bar{C}+\bar{l}_{\infty}+\bar{S}_{\infty}+\left(\bar{E}_{2}+\bar{E}_{4}+\cdots+\bar{E}_{2 m}\right)+\bar{D}_{1} \\
\bar{Z}= & 2 \bar{S}_{\infty}+(2 m+2)\left(\bar{l}_{\infty}+\bar{E}_{1}\right)+(2 m+3)\left(\bar{E}_{2}+\bar{E}_{3}\right)+\cdots \\
& +(3 m+2)\left(\bar{E}_{2 m}+\bar{E}_{2 m+1}\right)-\bar{D}_{2} .
\end{aligned}
$$




$$
\begin{aligned}
\bar{B}= & \bar{C}+\bar{l}_{\infty}+\bar{S}_{\infty}+\left(\bar{E}_{2}+\bar{E}_{4}+\cdots+\bar{E}_{b-a}\right)+\bar{D}_{1} \\
\bar{Z}= & 2 \bar{S}_{\infty}+\frac{b+1}{2}\left(\bar{l}_{\infty}+\bar{E}_{1}\right)+\frac{b+3}{2}\left(\bar{E}_{2}+\bar{E}_{3}\right)+\cdots \\
& +\frac{2 b-a-1}{2}\left(\bar{E}_{b-a-2}+\bar{E}_{b-a-1}\right)+\frac{2 b-a+1}{2} \bar{E}_{b-a}-\bar{D}_{2} .
\end{aligned}
$$

Case III. $c>a, c>b$

(III-1-1)

$$
\begin{aligned}
& \bar{B}=\bar{C}+\bar{l}_{\infty}+\bar{S}_{\infty}+\bar{E}_{1}+\bar{E}_{2}+\cdots+\bar{E}_{2 m}+\bar{D}_{1} \\
& \bar{Z}=2 \bar{S}_{\infty}+(3 m+1)\left(\bar{l}_{\infty}+\bar{E}_{1}+\bar{E}_{2}+\cdots+\bar{E}_{2 m}\right)-\bar{D}_{2} .
\end{aligned}
$$

(III-1-2)

$$
\begin{aligned}
& \bar{B}=\bar{C}+\bar{l}_{\infty}+\bar{S}_{\infty}+\bar{E}_{1}+\bar{E}_{2}+\cdots+\bar{E}_{2 m+1}+\bar{D}_{1} \\
& \bar{Z}=2 \bar{S}_{\infty}+(3 m+2)\left(\bar{l}_{\infty}+\bar{E}_{1}+\bar{E}_{2}+\cdots+\bar{E}_{2 m+1}\right)-\bar{D}_{2} .
\end{aligned}
$$

(III-1-3)

$$
\begin{aligned}
& \bar{B}=\bar{C}+\bar{l}_{\infty}+\bar{S}_{\infty}+\bar{E}_{1}+\bar{E}_{2}+\cdots+\bar{E}_{2 m+1}+\bar{D}_{1} \\
& \bar{Z}=2 \bar{S}_{\infty}+(3 m+3)\left(\bar{l}_{\infty}+\bar{E}_{1}+\bar{E}_{2}+\cdots+\bar{E}_{2 m+1}\right)+(3 m+4) \bar{E}_{2 m+2}-\bar{D}_{2} .
\end{aligned}
$$

$$
\begin{aligned}
\bar{B}= & \bar{C}+\bar{l}_{\infty}+\bar{S}_{\infty}+\bar{E}_{1}+\bar{E}_{2}+\cdots+\bar{E}_{c-b}+\bar{F}_{2}+\bar{F}_{4}+\cdots+\bar{F}_{6 m-c+1}+\bar{D}_{1} \\
\bar{Z}= & 2 \bar{S}_{\infty}+\frac{c+1}{2}\left(\bar{l}_{\infty}+\bar{E}_{1}+\bar{E}_{2}+\cdots+\bar{E}_{c-b}\right)+\frac{c+1}{2} \bar{F}_{1}+\frac{c+3}{2}\left(\bar{F}_{2}+\bar{F}_{3}\right)+\cdots \\
& +3 m\left(\bar{F}_{6 m-c-1}+\bar{F}_{6 m-c}\right)+(3 m+1) \bar{F}_{6 m-c+1}-\bar{D}_{2} .
\end{aligned}
$$

$$
\begin{aligned}
\bar{B}= & \bar{C}+\bar{l}_{\infty}+\bar{S}_{\infty}+\bar{E}_{1}+\bar{E}_{2}+\cdots+\bar{E}_{c-b}+\bar{F}_{2}+\bar{F}_{4}+\cdots+\bar{F}_{6 m-c+3}+\bar{D}_{1} \\
\bar{Z}= & 2 \bar{S}_{\infty}+\frac{c+1}{2}\left(\bar{l}_{\infty}+\bar{E}_{1}+\bar{E}_{2}+\cdots+\bar{E}_{c-b}\right)+\frac{c+1}{2} \bar{F}_{1}+\frac{c+3}{2}\left(\bar{F}_{2}+\bar{F}_{3}\right)+\cdots \\
& +(3 m+1)\left(\bar{F}_{6 m-c+1}+\bar{F}_{6 m-c+2}\right)+(3 m+2)\left(\bar{F}_{6 m-c+3}+\bar{F}_{6 m-c+4}\right)-\bar{D}_{2} .
\end{aligned}
$$

(III-3-1)

$$
\begin{aligned}
& \bar{B}=\bar{C}+T_{\infty}+\bar{S}_{\infty}+\bar{E}_{1}+\bar{E}_{2}+\cdots+\bar{E}_{c-b}+\bar{D}_{1} \\
& \bar{Z}=2 \bar{S}_{\infty}+\frac{c+1}{2}\left(\bar{l}_{\infty}+\bar{E}_{1}+\bar{E}_{2}+\cdots+\bar{E}_{c-b}\right)-\bar{D}_{2} .
\end{aligned}
$$


(III-3-2)

$$
\begin{aligned}
\bar{B}= & \bar{C}+\bar{l}_{\infty}+\bar{S}_{\infty}+\bar{E}_{1}+\bar{E}_{2}+\cdots+\bar{E}_{k}+\bar{D}_{1} \\
\bar{Z}= & 2 \bar{S}_{\infty}+\frac{c+1}{2}\left(\bar{l}_{\infty}+\bar{E}_{1}+\bar{E}_{2}+\cdots+\bar{E}_{(c-a) / 2}\right)+\frac{c-1}{2} \bar{E}_{(c-a) / 2+1}+\cdots \\
& +\frac{2 c-a-2 k+1}{2} \bar{E}_{k}-\bar{D}_{2},
\end{aligned}
$$

where $k$ is some positive integer.

(III-3-3)

$$
\begin{aligned}
\bar{B} & =\bar{C}+\bar{l}_{\infty}+\bar{S}_{\infty}+\bar{E}_{1}+\bar{E}_{2}+\cdots+\bar{E}_{c-b}+\bar{E}_{c-b+2}+\bar{E}_{c-b+4}+\cdots+\bar{E}_{b-a}+\bar{D}_{1} \\
\bar{Z} & =2 \bar{S}_{\infty}+\frac{c+1}{2}\left(T_{\infty}+\bar{E}_{1}+\bar{E}_{2}+\cdots+\bar{E}_{c-b}\right)+\frac{c-1}{2}\left(\bar{E}_{c-b+1}+\bar{E}_{c-b+2}\right) \\
& +\frac{c-3}{2}\left(\bar{E}_{c-b+3}+\bar{E}_{c-b+4}\right)+\cdots+\frac{a-2 b+2 c+1}{2}\left(\bar{E}_{b-a-1}+\bar{E}_{b-a}\right)-\bar{D}_{2} .
\end{aligned}
$$

Case IV. $a>b, a \geqslant c$

$$
\begin{aligned}
& \bar{B}=\bar{C}+\bar{l}_{\infty}+\bar{S}_{\infty}+\bar{E}_{1}+\bar{E}_{2}+\cdots+\bar{E}_{k}+\bar{D}_{1} \\
& \bar{Z}=2 \bar{S}_{\infty}+(m+1) \bar{l}_{\infty}+m \bar{E}_{1}+(m-1) \bar{E}_{2}+\cdots+(m+1-k) \bar{E}_{k}-\bar{D}_{2},
\end{aligned}
$$

where $k$ is some positive integer.

REMARK 2. In the above configurations of components and decompositions of $\left(\bar{\sigma}^{*} A\right)$ into $\bar{B}$ and $\bar{Z}$, we cannot explicitly determine the number of exceptional curves in Case (III-3-2) and Case IV. The following lemma enables us to deal with the situation:

LEMMA 3.4. Let $\tau=1 / t$ and $\xi=1 / x$. Suppose that the $C$ has a singularity at the point $\tau=\xi=0$ which is locally defined by the equation

$$
\eta^{2}=\tau\left(U(\tau, \xi) \xi^{2}+\tau^{s} V(\tau) \xi+\tau^{2 l} W(\tau)\right), \quad l>0, \quad s \geqslant 0,
$$

where either $U(\tau, \xi)=U_{0}(\tau) \in k[\tau]$ with $U_{0}(0) \neq 0$ or $U(\tau, \xi)=u_{2}(\tau) \xi^{2}+\tau^{h} u_{1}(\tau) \xi+u_{0}(\tau)$ with $u_{i}(\tau) \in k[\tau], u_{i}(0) \neq 0 \quad(i=0,1,2)$ and $h \geqslant s$, and where $V(\tau), W(\tau) \in k[\tau]$ with $V(0) \cdot W(0) \neq 0$. Then one can resolve its singularity by a finite succession of blowing-up at the singular point and its infinitely near singular points. Furthermore, the dual graph of the configuration of the exceptional divisors is as given in Figure 5, where $k$ is a positive integer and the components belonging to $\bar{B}$ in the decomposition $\left(\bar{\sigma}^{*} A\right)=\bar{B}-2 \bar{Z}$ are

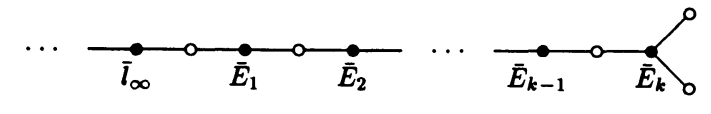

Figure 5. 
indicated as black vertices.

Proof. By blowing -up at $\tau=\xi=0$ and its infinitely near singular points, the lemma can be proved by induction on $s$.

Let $\sigma: F \rightarrow \bar{F}$ be the shortest succession of blowings-up of $\bar{F}$ such that if one writes $\left((\bar{\sigma} \sigma)^{*} A\right)$ in the form $\left((\bar{\sigma} \sigma)^{*} A\right)=B-2 Z$ with divisors $B$ and $Z$ uniquely determined as above, every irreducible component of $B$ is a connected component of $\operatorname{Supp}(B)$, that is, $\operatorname{Supp}(B)$ is nonsingular.

\section{LEMMA 3.5. Let $H$ be the normalization of $F$ in $\mathscr{K}$. Then $H$ is nonsingular.}

Proof. Let $\rho$ denote the normalization morphism $H \rightarrow F$. Let $Q$ be a point of $H$ and $P=\rho(Q) \in F$. If $\bar{\sigma} \sigma(P)$ is not a singular point of $C$, then $Q$ is a simple point by the same method as in $[7,(2.1)]$. Suppose $\bar{\sigma} \sigma(P)$ is a singular point of $C$ which lies on the fiber over $t=\infty$. Since we have the configuration of $(\bar{\sigma} \sigma)^{-1}\left(l_{\infty} \cup C \cup S_{\infty}\right)$ one can show by the same argument as in $[7,(2.6 .2)]$ that $Q$ is a simple point. The case where $\bar{\sigma} \sigma(P)$ is a singular point of $C \cap A_{k}^{2}=C \cap\left(F_{0}-\{x=0\} \cup\{t=\infty\}\right)$ can be treated similarly.

In subsequent arguments we need the following well-known lemma (cf. [1]).

Lemma 3.6. (1) Let $D_{1}, D_{2}$ be divisors on $F$. Then

$$
\left(\rho^{-1}\left(D_{1}\right) \cdot \rho^{-1}\left(D_{2}\right)\right)=2\left(D_{1} \cdot D_{2}\right) .
$$

(2) If $D$ is an irreducible curve contained in the support of $B$, then $\rho^{-1}(D)=2 \Delta$, where $\Delta$ is a nonsingular curve on $H$. Moreover, if $D \cong P_{k}^{1}$, then $\Delta \cong P_{k}^{1}$.

Now let $q:=p_{2} \bar{\sigma} \sigma \rho: H \rightarrow \boldsymbol{P}_{k}^{1}, \tilde{C}:=\sigma^{\prime}(\bar{C}), \tilde{S}_{\infty}:=\sigma^{\prime}\left(\bar{S}_{\infty}\right)$. Since $\bar{C}, \bar{S}_{\infty} \subset \operatorname{Supp}(\bar{B})$ we have $\tilde{C}, \tilde{S}_{\infty} \subset \operatorname{Supp}(B)$. Thus we can write $\rho^{-1}(\tilde{C})=2 \Gamma$ and $\rho^{-1}\left(\tilde{S}_{\infty}\right)=2 \Sigma_{\infty}$ with nonsingular curves $\Gamma$ and $\Sigma_{\infty}$ on $H$.

Let $l=\{t=\gamma\}$ be a general member of $\mathscr{F}$, and let $\tilde{l}=(\bar{\sigma} \sigma)^{\prime}(l)$. Then $\rho^{-1}(\tilde{l})-$ $\rho^{-1}(\tilde{l}) \cap \Sigma_{\infty}$ is easily shown to be isomorphic to a curve $y^{2}=A(x, \gamma)$ and $p_{a}\left(\rho^{-1}(\tilde{l})\right)=1$. Thus we obtain:

LEMMA 3.7. $q: H \rightarrow \boldsymbol{P}_{k}^{1}$ is a quasi-elliptic fibration with a regular cross-section $\Sigma_{\infty}$.

Let us illustrate the weighted graph of $q^{-1}(\infty) \cup \Sigma_{\infty}$. In Figure 6, a white (resp. black) vertex corresponds to a component of $q^{-1}(\infty) \cup \Sigma_{\infty}$ which is not contained in $\operatorname{Supp}(B)($ resp. contained in $\operatorname{Supp}(B))$. The weight at a vertex is the self-intersection number of the corresponding component. Two vertices are joined by a single (resp. double) edge if the corresponding components meet each other transversally at one point (resp. touch each other at one point with multiplicity 2). If omitted, the weight of a vertex is -2 .

Now contract all $(-1)$-curves contained in $q^{-1}(\infty)$ to obtain the minimal form of $q^{-1}(\infty)$ (Figure 7). Note that no $(-1)$-curves appear in Cases I and IV. 
Case I. $\quad a=b \geqslant c$

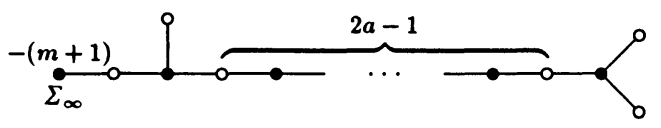

Case II. $\quad b>a, b \geqslant c$

(II-1)

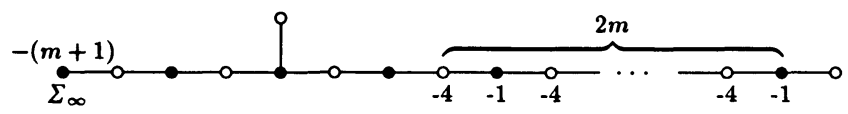

(II-2)

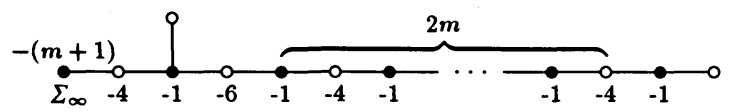

(II-3)

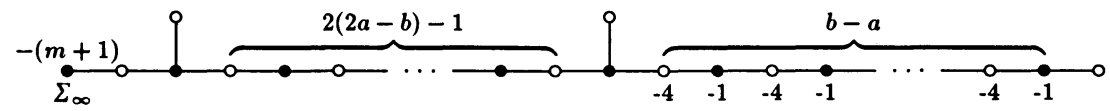

Case III. $c>a, c>b$

(III-1-1)

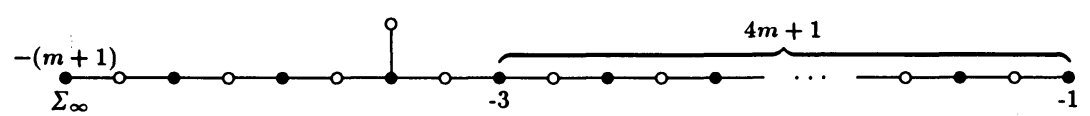

(III-1-2)

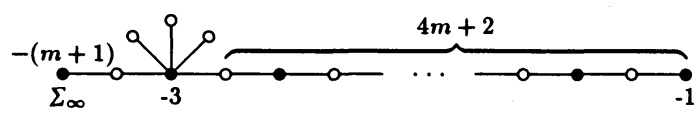

(III-1-3)

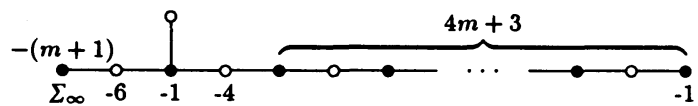


(III-2-1)

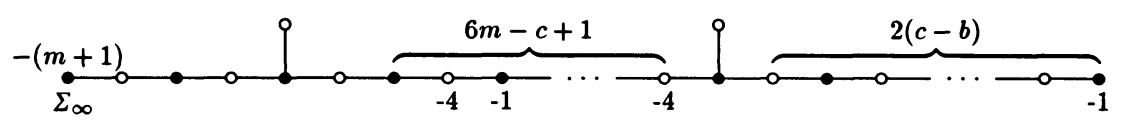

(III-2-2)

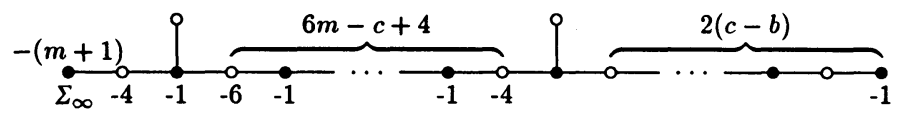

(III-3-1)

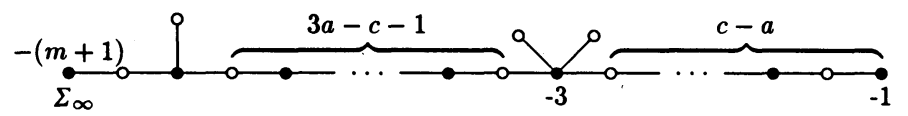

(III-3-2)

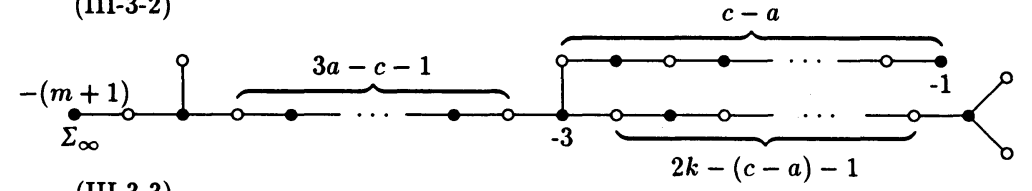

(III-3-3)

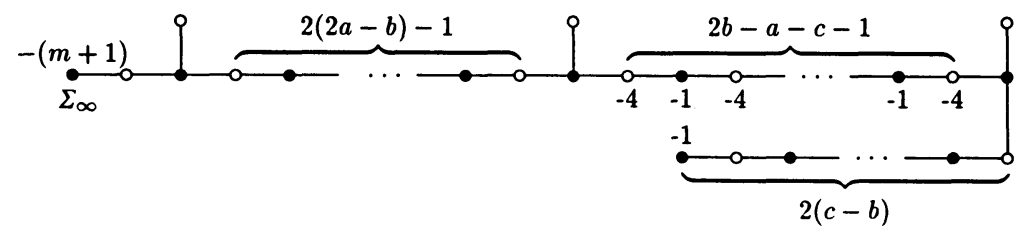

Case IV. $a>b, a \geqslant c$

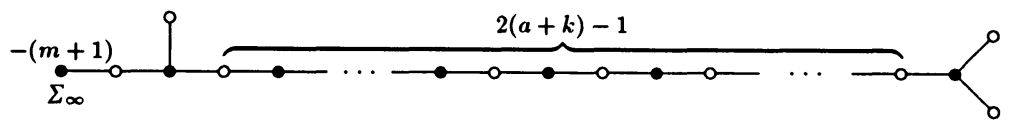

FIGURE 6.

To complete the proof of Theorem 1.1, we need the following lemma.

LEMMA 3.8. If $q^{-1}(\gamma)$ is a reducible singular fiber lying over the point $t=\gamma$ with $\gamma \neq \infty$, then $\gamma$ is a solution of $\Delta(t)=0$, and the contraction of the $(-1)$-curves contained in $q^{-1}(\gamma)$ does not affect the self-intersection number of $\Sigma_{\infty}$.

Proof. We may assume that $C$ has a singularity at the point $x=t=0$ after a change of variables $(x, t) \mapsto\left(x+\beta(\gamma)^{1 / 2}, t+\gamma\right)$, and that $C$ is defined locally by the equation $x^{3}+\alpha(t) x^{2}+\beta(t) x+\gamma(t)=0$ with $t$ dividing $\alpha(t), \beta(t)$ and $\gamma(t)$. Then, blowingup the singular point $x=t=0$ and its infinitely near singular points, taking the normalization of $F_{0}$ in $\mathscr{K}$ as before and contracting all (-1)-curves in the fiber of $q$ so obtained, one can easily find that the component of the singular fiber of $q$ over $t=0$ 
Case II. $\quad b>a, b \geqslant c$

(II-1)

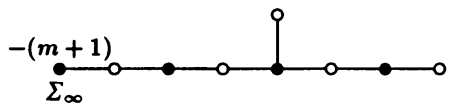

$$
\underset{\sum_{\infty}}{-(m+1)}
$$

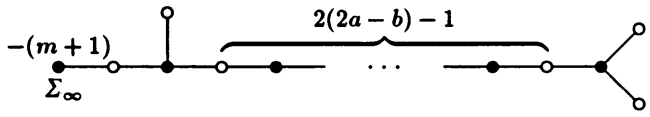

Case III. $c>a, c>b$

(III-1-1)

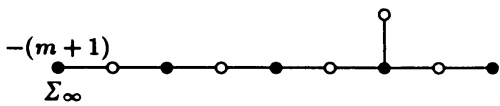

(III-2-1)

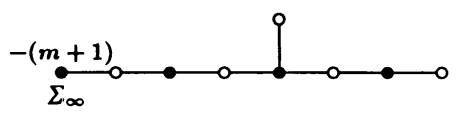

(III-3-1)
(III-1-2)

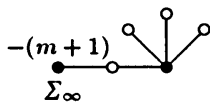

(III-2-2)

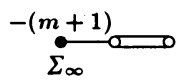

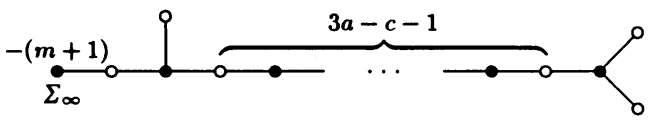

(III-3-2)

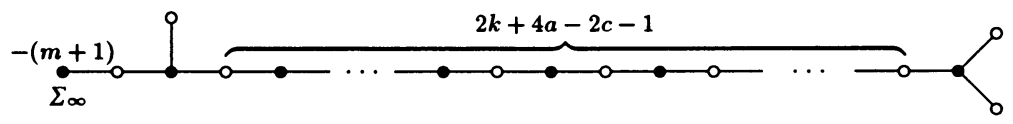

(III-3-3)

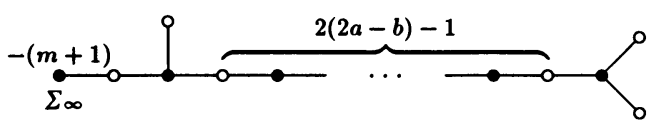

Figure 7.

which intersects $\Sigma_{\infty}$ cannot be contracted by the blowing-down of all (-1)-curves in $q^{-1}(0)$. We leave the details to the reader. See also [7].

Therefore we have $\left(\Sigma_{\infty}^{2}\right)=-(m+1)$.

Let $\hat{q}: \hat{H} \rightarrow P_{k}^{1}$ be the quasi-elliptic fibration which is obtained from $q: H \rightarrow \boldsymbol{P}_{k}^{1}$ by contracting all $(-1)$-curves contained in the fibers. Let $\hat{\Sigma}_{\infty}$ denote the proper transform of $\Sigma_{\infty}$ under this contraction. Lemma 3.8 thus means that $\left(\hat{\Sigma}_{\infty}^{2}\right)=-(m+1)$. 
Meanwhile, the canonical divisor formula for a quasi-elliptic fibration (see, e.g., [3]) implies

$$
K_{\hat{\mathbf{H}}} \sim\left(\chi\left(\mathcal{O}_{\hat{\mathbf{H}}}\right)-2\right) F,
$$

where $F$ is a general fiber of $\hat{q}$. Since $\hat{\Sigma}_{\infty}$ is a cross-section of $\hat{q}, \hat{\Sigma}_{\infty}$ is isomorphic to $\boldsymbol{P}_{\boldsymbol{k}}^{1}$. Then, applying the arithmetic genus formula to $\hat{\Sigma}_{\infty}$, we obtain

$$
\left(\chi\left(\mathcal{O}_{\hat{H}}\right)-2\right)\left(F \cdot \hat{\Sigma}_{\infty}\right)+\left(\hat{\Sigma}_{\infty}^{2}\right)=-2,
$$

whence $\chi\left(\mathcal{O}_{\hat{H}}\right)=m+1$. By virtue of the arguments in [7], we then complete the proof of Theorem 1.1.

Let $\mathscr{L}=R^{1} \hat{q}_{*} \mathcal{O}_{\hat{\mathbf{H}}}$. Then $\operatorname{deg} \mathscr{L}=-\chi\left(\mathcal{O}_{\hat{\mathbf{A}}}\right)=-(m+1)$. Hence $\operatorname{deg}\left(\mathscr{L}^{\otimes(-12)} \otimes \omega_{\mathbf{P}^{1}}^{\otimes 2}\right)=$ $12(m+1)-4=12 m+8$. By the remark given before Lemma 3.1, we have

$$
\Delta_{\infty}(\tau)=\tau^{12 m+8} \Delta\left(\frac{1}{\tau}\right) .
$$

This implies that $\left\{\Delta(t), \Delta_{\infty}(\tau)\right\}$ gives rise to a section of $\Gamma\left(\boldsymbol{P}_{k}^{1}, \mathscr{L}^{\otimes(-12)} \otimes \omega_{\boldsymbol{P}^{1}}^{\otimes 2}\right)$, which we call the discriminant of the quasi-elliptic fibration $\hat{q}: \hat{H} \rightarrow \boldsymbol{P}_{\boldsymbol{k}}^{1}$.

Thus we have an equality

$$
12 \chi\left(\mathcal{O}_{\hat{\mathrm{H}}}\right)-4=\operatorname{deg}(\Delta),
$$

where $(\Delta)$ is the divisor on $\boldsymbol{P}^{1}$ corresponding to the section $\left\{\Delta(t), \Delta_{\infty}(\tau)\right\}$ of $\Gamma\left(\boldsymbol{P}_{k}^{1}\right.$, $\left.\mathscr{L}^{\otimes(-12)} \otimes \omega_{\mathbf{P}^{1}}^{\otimes 2}\right)$.

4. Reducible singular fibers and the torsion-rank. In this section, we consider reducible singular fibers which appear in a quasi-elliptic fibration in characteristic 2 and give the conditions for a combination of reducible singular fibers to exhaust all degenerate fibers of a quasi-elliptic fibration.

To analyze the reducible singular fibers, we consider a Weierstrass equation in the local case, that is, $C=\operatorname{spec} k[[t]]$.

LEMMA 4.1. Let $f: X \rightarrow C \cong \operatorname{Spec} k[[t]]$ be a quasi-elliptic surface with a section defined by

$$
y^{2}=x^{3}+\varphi(t) x+\psi(t)
$$

with $\varphi(t), \psi(t) \in k[[t]]$ and either $\varphi(t) \notin k[[t]]^{2}$ or $\psi(t) \notin k[[t]]^{2}$. Then the equation of $X$ can be put into a form

$$
y^{2}=x^{3}+\left(\alpha^{4} t^{2 s}+\beta^{2} t^{m}\right) x+\gamma^{2} t^{k}
$$

such that

$$
\beta \neq 0 \quad \text { or } \quad \gamma \neq 0,
$$




$$
s=1 \text { or } 1 \leqslant m \leqslant 3 \text { or } 1 \leqslant k \leqslant 5,
$$

where each of $\alpha, \beta, \gamma$ is either a unit in $k[[t]]$ or a zero, and each of $s, m, k$ is a positive odd integer (resp. zero) if the corresponding coefficient $\alpha^{4}, \beta^{2}, \gamma^{2}$ is not equal to zero (resp. is equal to zero).

Proof. By Lemma 3.1 we may assume that $\varphi(t)$ and $\psi(t)$ satisfy the conditions (1), (2), (3) in Theorem 1.1. Note that Lemma 3.1 holds also for $k[[t]]$ instead of $k[t]$. Hence we can write

$$
\varphi(t)=\sum_{i \geqslant 0} \alpha_{i} t^{4 i+2}+\sum_{j \geqslant 0} \beta_{j} t^{2 j+1}, \quad \psi(t)=\sum_{k \geqslant 0} \gamma_{k} t^{2 k+1} .
$$

Let $i_{0}:=\min \left\{i\right.$ with $\left.\alpha_{i} \neq 0\right\}$ if $\alpha_{i} \neq 0$ for some $i$ and $i_{0}=-1$ otherwise; let $j_{0}:=\min \{j$ with $\left.\beta_{j} \neq 0\right\}$ if $\beta_{j} \neq 0$ for some $j$ and $j_{0}=-1$ otherwise; let $k_{0}:=\min \left\{k\right.$ with $\left.\gamma_{k} \neq 0\right\}$ if $\gamma_{k} \neq 0$ for some $k$ and $k_{0}=-1$ otherwise.

We set $\alpha=\alpha_{i_{0}}^{\prime}+\alpha_{i_{0}+1}^{\prime} t+\cdots$ and $s=2 i_{0}+1$ if $i_{0} \geqslant 0, \beta=\beta_{j_{0}}^{\prime}+\beta_{j_{0}+1}^{\prime} t+\cdots$ and $m=2 j_{0}+1$ if $j_{0} \geqslant 0, \gamma=\gamma_{k_{0}}^{\prime}+\gamma_{k_{0}+1}^{\prime} t+\cdots$, and $k=2 k_{0}+1$ if $k_{0} \geqslant 0$, where $\alpha_{i}^{\prime}=\alpha_{i}^{1 / 4}$, $\beta_{j}^{\prime}=\beta_{j}^{1 / 4}$ and $\gamma_{k}^{\prime}=\gamma_{k}^{1 / 4}$. Otherwise, we set $\alpha=s=0$ if $i_{0}=-1, \beta=m=0$ if $j_{0}=-1$ or $\gamma=k=0$ if $k_{0}=-1$, respectively. Then the conditions of the assertion are satisfied in view of Lemma 3.1.

Using this local Weierstrass equation, we can classify the reducible singular fibers as follows:

Proposition 4.2 (cf. [4, (5.5.10)]). Let $f: X \rightarrow C$ be a quasi-elliptic surface defined by (4.1), (4.2) and (4.3) in Lemma 4.1. Then the fiber over $t=0$ is determined as

$\begin{array}{lccc}\text { (1) } m \geqslant k \neq 0 \text { or } m=0 \\ \text { type } & v(\Delta) & k & s \\ \text { II } & 0 & 1 & \geqslant 0 \\ \text { I }_{0}^{*} & 4 & 3 & \geqslant 0 \\ \text { II }^{*} & 8 & 5 & \neq 1 \\ \text { I }_{2 k-6}^{*} & 2 k-2 & \geqslant 5 & 1\end{array}$

(2) $k>m \neq 0$ or $k=0$

$\begin{array}{lccc}\text { type } & v(\Delta) & m & s \\ \text { III } & 1 & 1 & \geqslant 0 \\ \text { III* } & 7 & 3 & \neq 1 \\ \text { I }_{2 m-4}^{*} & 2 m & \geqslant 3 & 1\end{array}$

Here $v$ denotes the valuation of $k[[t]]$ with $v(t)=1$ and $\Delta$ stands for the polynomial $\Delta(t)$.

Now, let us return to the global situation, i.e., $C$ is the projective line $\boldsymbol{P}^{1}$. By the above classification, we know all possible types of the reducible singular fibers of $X$, and we have relations among the Euler-Poincare characteristic, the number of the reducible singular fibers of respective types and the torsion-rank. Let $v(S)$ denote the number of singular fibers of type $S$ (cf. the classification by Kodaira [6]). Then, from the equality (3.5) and Proposition 4.2, we have:

Proposition 4.3. Let $f: X \rightarrow C$ be a unirational quasi-elliptic surface with section 
in characteristic 2 . Then

$$
12 \chi-4=\sum_{k \geqslant 0}(2 k+4) v\left(\mathrm{I}_{2 k}^{*}\right)+8 v\left(\mathrm{II}^{*}\right)+v(\mathrm{III})+7 v\left(\mathrm{III}^{*}\right),
$$

where $\chi$ is the Euler-Poincaré characteristic of $X$.

Let $T$ be the trivial lattice generated by the zero section and all the irreducible components of the fibers. We have a natural decomposition

$$
T=\langle O, F\rangle \oplus\left(\bigoplus_{v \in R} T_{v}\right),
$$

where $T_{v}$ is the lattice generated by all the irreducible components of $f^{-1}(v)$ except the irreducible component meeting the zero section $(O)$. Hence,

$$
\operatorname{det} T=-\prod_{v \in R} \operatorname{det} T_{v}=-2 \sum_{k \geqslant 0} 2 v\left(\mathrm{I}_{2 k}^{*}\right)+v(\mathrm{III})+v(\mathrm{III}),
$$

since the lattice $T_{v}$ for the fiber of type II* is unimodular. Note that the exponent of $\operatorname{det} T$ is an even integer by (4.4). By (2.1), we have

$$
\operatorname{det} \operatorname{NS}(X)=\operatorname{det} T /|E(K)|^{2} \text {. }
$$

Since the right hand side of (4.5) has even exponent as remarked above, we can write

$$
\operatorname{det} \operatorname{NS}(X)=-2^{2 \sigma_{0}} \quad \text { with } \quad \sigma_{0} \in Z, \quad \sigma_{0} \geqslant 0 .
$$

By comparison of the exponents of (4.5) and (4.6), we have:

Proposition 4.4. Let the notation be as in Proposition 4.3. Then

$$
\sigma_{0}+\dot{r}=\frac{1}{2}\left\{\sum_{k \geqslant 0} 2 v\left(\mathrm{I}_{2 k}^{*}\right)+v(\mathrm{III})+v\left(\mathrm{III}^{*}\right)\right\},
$$

where $r$ is the torsion-rank of the Mordell-Weil group (cf. the Introduction).

ReMarK 3. The above invariant $\sigma_{0}$ is called the Artin invariant when $X$ is a supersingular $K 3$ surface and takes a value between 1 and 10 . It plays a very important role in the theory of moduli space of supersingular $K 3$ surfaces (cf. Artin [2]).

Proposition 4.5. For a section $P(\neq O)$, we have

$$
2 \chi+2(P \cdot O) \leqslant \frac{1}{2} v(\mathrm{III})+\frac{3}{2} v\left(\mathrm{III}^{*}\right)+\sum_{b \geqslant 0}\left(1+\frac{b}{4}\right) v\left(\mathrm{I}_{b}^{*}\right) .
$$

Proof. For a torsion point $P \in E(K)$, we have $\langle P, P\rangle=0$ where $\langle$,$\rangle is the height$ pairing on $E(K)$ defined in Section 2 (cf. Shioda [9]). Thus, the assertion is a consequence of (2.2) and straightforward computations of $\operatorname{Contr}_{v}(P)$.

In the next section, we use this proposition to check whether two sections are 
intersect each other.

5. Rational quasi-elliptic surfaces. In this section, we study the relationship between reducible singular fibers of rational quasi-elliptic fibrations and their MordellWeil groups and look into the configurations of sections and reducible singular fibers on rational quasi-elliptic surfaces. Let $f: X \rightarrow \boldsymbol{P}^{1}$ be a rational quasi-elliptic surface with a section $O$. Then by virtue of Theorem 1.1 we may start with an affine expression for $X$ :

$$
y^{2}=x^{3}+\varphi(t) x+\psi(t), \quad \varphi(t)=a_{3} t^{3}+a_{2} t^{2}+a_{1} t, \quad \psi(t)=b_{5} t^{5}+b_{3} t^{3}+b_{1} t,
$$

where $\varphi(t) \notin k[t]^{2}$ or $\psi(t) \notin k[t]^{2}$. To determine the reducible singular fibers, we look as the discriminant $\Delta(t)$ of $X$, given as

$$
\begin{aligned}
\Delta(t) & =\varphi(t)\left(\varphi^{\prime}(t)\right)^{2}+\left(\psi^{\prime}(t)\right)^{2} \\
& =b_{5}^{2} t^{8}+a_{3}^{3} t^{7}+a_{2} a_{3}^{2} t^{6}+a_{1} a_{3}^{2} t^{5}+b_{3}^{2} t^{4}+a_{1}^{2} a_{3} t^{3}+a_{1}^{2} a_{2} t^{2}+a_{1}^{3} t+b_{1}^{2} .
\end{aligned}
$$

Set

$$
d_{1}:=a_{1}^{2} b_{5}+a_{1} a_{3} b_{3}+a_{3}^{2} b_{1}, \quad d_{2}:=a_{1} a_{2} a_{3}+b_{3}^{2}, \quad d_{3}:=a_{2}^{2}+a_{1} a_{3} .
$$

The following formulas suggest how $d_{1}, d_{2}$ and $d_{3}$ are incorporated into concrete computations:

(1) $\Delta^{\prime}(t)=\left(a_{3} t^{2}+a_{1}\right)^{3}=\varphi^{\prime}(t)^{3}$.

(2) $\Delta\left(\sqrt{a_{1} / a_{3}}\right)=d_{1}^{2} / a_{3}^{4}=\psi^{\prime}\left(\sqrt{a_{1} / a_{3}}\right)^{2}$, if $a_{3} \neq 0$.

(3) $\Delta(t)=\left(t^{2}+a_{1} / a_{3}\right)^{2} \Delta_{1}(t)+a_{3}^{3} t\left(t^{2}+a_{1} / a_{3}\right)^{3}, \Delta_{1}(t)=b_{5}^{2} t^{4}+a_{2} a_{3}^{2} t^{2}+b_{3}^{2}$ $+a_{1}^{2} b_{5}^{2} / a_{3}^{2}, \Delta_{1}\left(\sqrt{a_{1} / a_{3}}\right)=d_{2}$, if $d_{1}=0$.

(4) $\Delta(t)=\left(t^{2}+a_{1} / a_{3}\right)^{3} \Delta_{2}(t), \Delta_{2}(t)=b_{5}^{2} t^{2}+a_{3}^{3} t+a_{2} a_{3}^{2}+a_{1} b_{5}^{2} / a_{3}$, if $d_{1}=d_{2}=0$. Moreover $\Delta_{2}\left(\sqrt{a_{1} / a_{3}}\right)=0$ if and only if $d_{3}=0$.

By virtue of Proposition 4.3 we have a list of all the possible types of reducible singular fibers in the case of rational quasi-elliptic surfaces:

Proposition 5.1. There are seven possible patterns of reducible singular fibers in terms of their types:
(a) one II*,
(b) one $\mathrm{I}_{4}^{*}$,
(c) one III and one III*,
(d) two I⿳亠丷厂 's,
(e) one I2 and two III's,
(f) one I* and four III's,
(g) eight III's.

Proof. Since $X$ is assumed to be rational, we have $\chi=1$. Hence the right hand side equals 8 in the formula in Proposition 4.3. Now it is easy to list up all possibilities. 
TABLE 1 .

\begin{tabular}{|c|c|c|c|}
\hline $\begin{array}{l}\text { The pattern of } \\
\text { reducible fibers. }\end{array}$ & The defining equation. & $r$ & nonzero section \\
\hline (a) & $y^{2}=x^{3}+t^{5}$ & 0 & \\
\hline (b) & $y^{2}=x^{3}+t^{2} x+t^{5}$ & 1 & $\left(t^{2}+t, t^{3}\right)$ \\
\hline (c) & $y^{2}=x^{3}+t^{3} x$ & 1 & $(0,0)$ \\
\hline (d) & $\begin{array}{l}y^{2}=x^{3}+a t^{2} x+t^{3} \\
\text { with } a \in k\end{array}$ & 2 & $(u t, 0) \quad$ with $u^{3}+a u+1=0$ \\
\hline (e) & $y^{2}=x^{3}+\left(t^{3}+t\right) x$ & 2 & $\begin{array}{l}(0,0) \\
\left(t+1, t^{2}+1\right) \\
\left(t^{2}+t, t^{3}+t\right)\end{array}$ \\
\hline (f) & $\begin{array}{l}y^{2}=x^{3}+\left(t^{3}+a t^{2}+t\right) x \\
\text { with } a \in k^{*}\end{array}$ & 3 & $\begin{array}{l}(0,0) \\
\left(a^{1 / 2} t, a^{1 / 4}\left(t^{2}+t\right)\right) \\
\left(a^{-1 / 2}\left(t^{2}+a t+1\right), a^{-3 / 4}\left(t^{3}+(a+1) t^{2}+(a+1) t+1\right)\right) \\
\left(u^{-1} t^{2}+u t, u^{-3 / 2}\left(t^{3}+a^{1 / 2} u t^{2}+u^{2} t\right)\right) \\
\left(u t+u^{-1}, u^{1 / 2}\left(t^{2}+a^{1 / 2} u^{-1} t+u^{-2}\right)\right) \\
\quad \text { with } u^{2}+a^{1 / 2} u+1=0\end{array}$ \\
\hline (g) & $\begin{array}{l}y^{2}=x^{3}+\left(t^{3}+a t^{2}+b t\right) x+t^{3} \\
\text { with } a \in k \text { and } b \in k^{*}\end{array}$ & 4 & $\begin{array}{l}\left(u t, u^{1 / 2}\left(t^{2}+b^{1 / 2} t\right)\right) \\
\left(u^{-1} t^{2}+u t+b u^{-1}, u^{-3 / 2}\left(t^{3}+\left(b+a u^{2}\right)^{1 / 2} t^{2}\right.\right. \\
\left.\left.\quad+\left(b^{2}+a b u^{2}\right) t+b^{3 / 2}\right)\right) \text { with } u^{3}+a u+1=0 \\
\left(v^{-1} t^{2}+v t, v^{-3 / 2}\left(t^{3}+a^{1 / 2} v t^{2}+b^{1 / 2} v^{2} t\right)\right) \\
\left(v t+b v^{-1}, v^{-3 / 2}\left(v^{2} t^{2}+a^{1 / 2} b^{1 / 2} v t+b^{3 / 2}\right)\right) \\
\quad \text { with } v^{4}+a v^{2}+v+b=0 \\
\left(t^{2} /\left(t^{2}+b\right),\left(a^{1 / 2} t^{4}+t^{3}+a^{1 / 2} b t^{2}\right) /\left(t+b^{1 / 2}\right)^{3}\right)\end{array}$ \\
\hline
\end{tabular}

Now we state a classification theorem by means of the given equation for $X$.

THEOREM 5.2. (1) The types of reducible singular fibers are classified into seven cases (a)-(g) as follows:

(i) Suppose $\varphi \in K^{2}$. Then necessarily have $d_{1}=0$. If $d_{2}=d_{3}=0$ then the type is (a); if $d_{2}=0$ and $d_{3} \neq 0$ then the type is $(\mathrm{b}) ;$ if $d_{2} \neq 0$ then the type is (d).

(ii) Suppose $\varphi \notin K^{2}$. If $d_{1}=d_{2}=d_{3}=0$ then the type is (c); if $d_{1}=d_{2}=0$ and $d_{3} \neq 0$ then the type is (e); if $d_{1}=0$ and $d_{2} \neq 0$ then the type is (f); if $d_{1} \neq 0$ then the type is $(\mathrm{g})$.

(2) The torsion-rank is determined uniquely by the type of reducible singular fibers as in Table 1.

Proof. (1) To determine the type of the reducible singular fibers, we look at the discriminant divisor $\Delta$ on $\boldsymbol{P}^{1}$ which corresponds to the polynomial $\Delta(t)$ (cf. (5.2)). By Proposition 4.2, one can find out easily that the possible types of the divisor $\Delta$ are 
as follows:
( $\left.T_{1}\right) \quad 8 P_{1}$
$\cdots$ (a), (b)
( $\left.T_{2}\right) 7 P_{1}+P_{2}$
( $\left.T_{3}\right) 4 P_{1}+4 P_{2}$
( $\left.T_{4}\right) 6 P_{1}+P_{2}+P_{3}$
( $\left.T_{5}\right) \quad 4 P_{1}+P_{2}+P_{3}+P_{4}+P_{5}$
( $\left.T_{6}\right) \quad P_{1}+P_{2}+\cdots+P_{8}$

where $P_{1}, P_{2}, \ldots, P_{8}$ are distinct points of $\boldsymbol{P}^{1}$.

We start from the equation given in (5.1). We consider also the equation

$$
\eta^{2}=\xi^{3}+\left(a_{1} \tau^{3}+a_{2} \tau^{2}+a_{3} \tau\right) \xi+\left(b_{1} \tau^{5}+b_{3} \tau^{3}+b_{5} \tau\right) .
$$

(See the paragraph below Lemma 3.1 in Section 3.) Moreover, note that $b_{1}, b_{3}, b_{5}$ cannot be simultaneously zero, for otherwise $X$ is singular.

(i) Suppose first $\varphi \in K^{2}$, i.e., $a_{1}=a_{3}=0$. Then $\varphi^{\prime}=0$ and $\psi \neq 0$. Thus $d_{1}=0$ and $\Delta(t)=\psi^{\prime}(t)^{2}=\left(b_{5}^{1 / 2} t^{2}+b_{3}^{1 / 3} t+b_{1}^{1 / 2}\right)^{4}$. Clearly the type of $\Delta$ is $\left(T_{1}\right)$ if and only if $b_{3}=0$ while the type of $\Delta$ is $\left(T_{3}\right)$ if and only if $b_{3} \neq 0$. If $b_{3}=0$, then $\Delta=8 P_{1}$. Hence the reducible singular fiber over $P_{1}$ is II* if $a_{2}=0$ and $\mathrm{I}_{4}^{*}$ if $a_{2} \neq 0$ by Proposition 4.2. Here note that $d_{2}=b_{3}^{2}$ and $d_{3}=a_{2}^{2}$ in this case. If $b_{3} \neq 0$, then $\Delta=4 P_{1}+4 P_{2}$ and the reducible singular fibers over $P_{1}$ and $P_{2}$ are of type $\mathrm{I}_{0}^{*}$.

(ii) Next, suppose $\varphi \notin K^{2}$. The possibilities for the type of $\Delta$ are $\left(T_{2}\right),\left(T_{4}\right),\left(T_{5}\right)$ and $\left(T_{6}\right)$. We can show that $d_{1} \neq 0$ if and only if the roots of $\Delta(t)=0$ are all simple. Hence, if $d_{1} \neq 0$ the type of $\Delta$ is $\left(T_{6}\right)$. By the formula given before Proposition 5.1, the type of $\Delta$ is $\left(T_{5}\right)$ if $d_{1}=0$ and $d_{2} \neq 0,\left(T_{4}\right)$ if $d_{1}=d_{2}=0$ and $d_{3} \neq 0$, and $\left(T_{2}\right)$ if $d_{1}=d_{2}=d_{3}=0$.

(2) When we pick up one standard form among the rational quasi-elliptic surfaces having the same fiber type, we make frequent use of the following lemma.

\section{Lemma 5.3. Suppose $X$ is defined by an affine equation}

$$
y^{2}=x^{3}+\left(\varphi_{0}(t)+a_{k} t^{k}\right) x+\left(\psi_{0}(t)+b_{l} t^{l}\right)
$$

with $\varphi_{0}(t), \psi_{0}(t) \in k[t]$ and $a_{k} \cdot b_{l} \neq 0$. If $2 l \neq 3 k$, then $X$ is isomorphic to the one defined by the same equation as (5.3) with $a_{k}=b_{l}=1$.

For the proof of this lemma, one can easily check that two surfaces given in the statement are isomorphic to each other by a suitable change of variables as in $[5$, Lemma 3.6].

The calculation of rational points in Table 1 is based on the method to be explained in Section 6. The last statement of the theorem is clear by Proposition 4.4, since $\sigma_{0}=0$ for rational surfaces.

In the rest of this section, we describe how the reducible singular fibers and sections intersect each other for each of the types classified in Theorem 5.2. For each of the 
type (a)

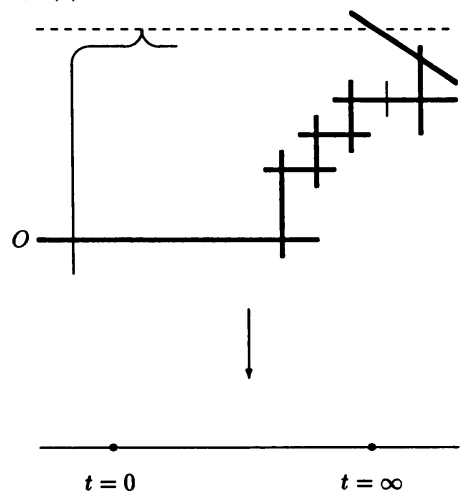

type (c)
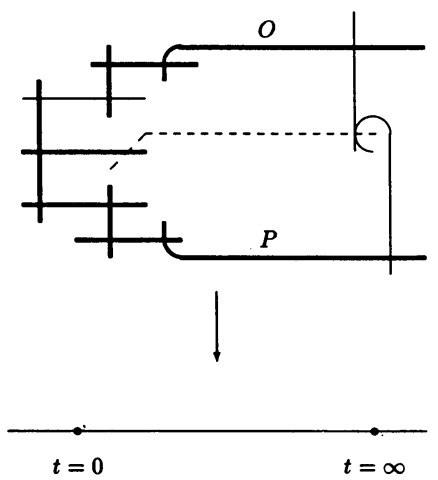

type (b)
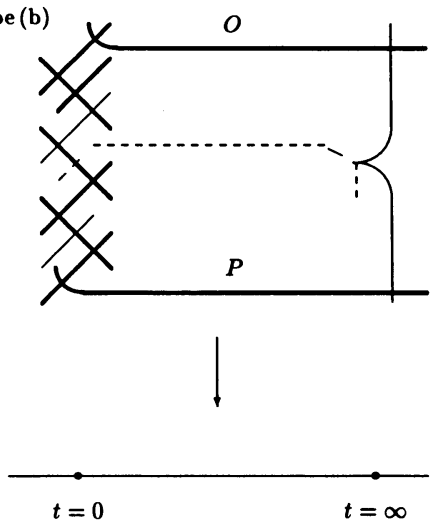

type (d)
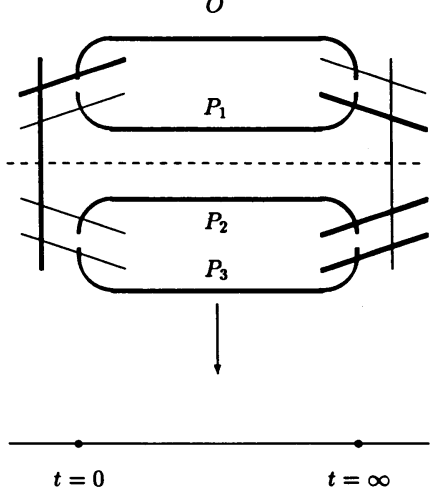

type (e)
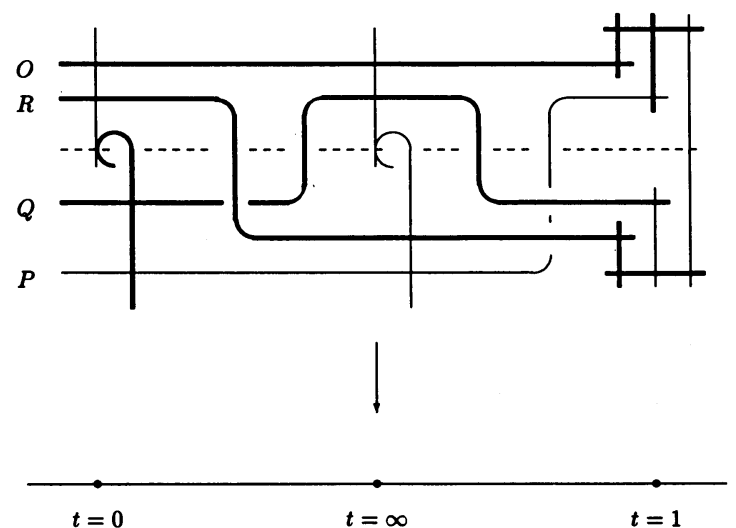

FIGURE 8. 

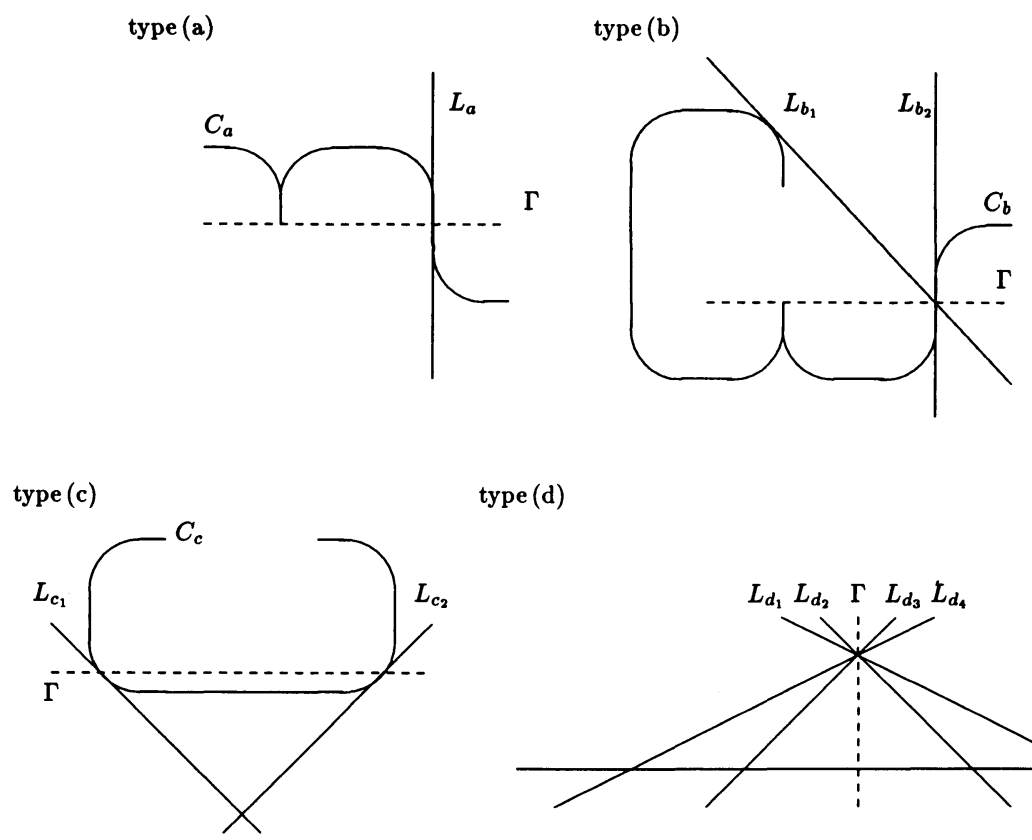

type (d)

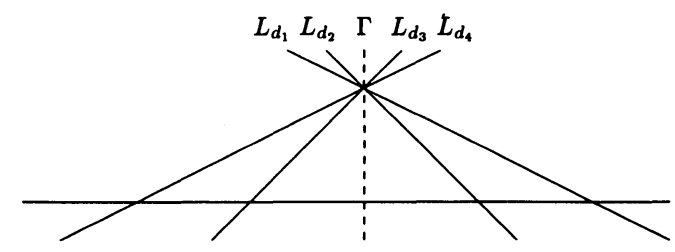

FIGURE 9.

types (a), (b), (c), (d) and (e) in Table 1, the configurations are simple (cf. Figure 8), and we can easily find out how they are constructed from $\boldsymbol{P}^{2}$ by blowing up nine points.

REMARK 4. By the blowing-down of the nine $\boldsymbol{P}^{1}$ 's which are drawn as bold lines in each figure, one can obtain the configuration of curves in $\boldsymbol{P}^{\mathbf{2}}$ for each of the types (a), (b), (c) and (d) as in Figure 9, where $C_{a}, C_{b}$ are curves of degree 3 with cusp, $C_{c}$ is a conic, $L_{*}$ and $\Gamma$ are lines, and the intersection of these curves are described as

$$
\left(C_{b} \cdot L_{b_{1}}\right)=\left(C_{c} \cdot L_{c_{1}}\right)=\left(C_{c} \cdot L_{c_{2}}\right)=2, \quad\left(C_{a} \cdot L_{a}\right)=\left(C_{b} \cdot L_{b_{2}}\right)=3 .
$$

In the case of the type (e), we can easily check that we obtain a configuration of four lines, a conic and a cubic with cusp in $\boldsymbol{P}^{2}$, which we do not give here.

The cases (f) and (g) are more complicated. In the case (f), the reducible singular fibers are of type III over $t=0, \infty, \alpha_{1}, \alpha_{2}$, where $\alpha_{1}$ and $\alpha_{2}$ are two solutions of the equation $t^{2}+a t+1=0$ with $a \neq 0$ and $\mathrm{I}_{0}$ over $t=1$. We name their components as in Figure 10.

Then the configuration is as in Table 2 .

In Table 2, $P_{1}=(0,0), P_{2}=\left(a^{1 / 2}, a^{1 / 4}\left(t^{2}+t\right)\right), P_{3}=\left(a^{-1 / 2}\left(t^{2}+a t+1\right), a^{-3 / 4}\left(t^{3}+\right.\right.$ $\left.\left.(a+1) t^{2}+(a+1) t+1\right)\right), \quad Q_{i}=\left(u_{i}^{-1} t^{2}+u_{i} t, \quad u_{i}^{-3 / 2}\left(t^{3}+a^{1 / 2} u_{i} t^{2}+u_{i}^{2} t\right)\right), \quad R_{i}=\left(u_{i} t+u_{i}^{-1}\right.$, $\left.u_{i}^{1 / 2}\left(t^{2}+a^{1 / 2} u_{i}^{-1} t+u_{i}^{-2}\right)\right)(i=1,2)$ are all sections, where $u_{i}(i=1,2)$ are the two roots of the equation $u^{2}+a^{1 / 2} u+1=0$. 

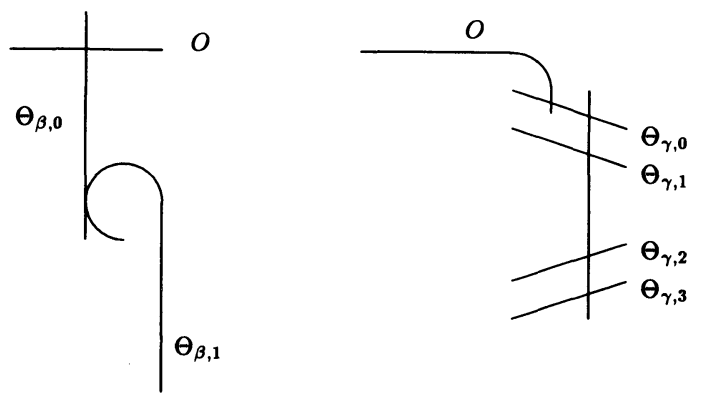

Figure 10.

TABLe 2 .

\begin{tabular}{|l|c|c|c|c|}
\hline & $\beta=0$ & $\beta=\infty$ & $\beta=\alpha_{1}$ & $\beta=\alpha_{2}$ \\
\hline Sections intersecting & $O, R_{2}$ & $O, Q_{2}$ & $O, Q_{2}$ & $O, R_{3}$ \\
$\Theta_{\beta, 0}$ & $R_{1}, P_{3}$ & $P_{3}, Q_{1}$ & $R_{1}, P_{2}$ & $P_{2}, Q_{1}$ \\
\hline Sections intersecting & $P_{1}, Q_{2}$ & $P_{1}, R_{2}$ & $P_{1}, R_{2}$ & $P_{1}, Q_{2}$ \\
$\Theta_{\beta, 1}$ & $P_{2}, Q_{1}$ & $R_{1}, P_{2}$ & $P_{3}, Q_{1}$ & $R_{1}, P_{3}$ \\
\hline
\end{tabular}

\begin{tabular}{|l|c|}
\hline & $\gamma=1$ \\
\hline Sections intersecting $\Theta_{\gamma, 0}$ & $O, P_{1}$ \\
\hline Sections intersecting $\Theta_{\gamma, 1}$ & $Q_{2}, R_{3}$ \\
\hline Sections intersecting $\Theta_{\gamma, 2}$ & $P_{2}, R_{1}$ \\
\hline Sections intersecting $\Theta_{\gamma, 3}$ & $Q_{1}, P_{3}$ \\
\hline
\end{tabular}

EXAMPLE 1. After blowing down the sections and irreducible components of the fibers (for example, $Q_{1}, R_{1}, Q_{2}, R_{2}, P_{2}, P_{3}, P_{1}, \Theta_{1,1}, \Theta_{1,4}$ in this order), one can obtain a configuration of curves in $\boldsymbol{P}^{2}$ which consist of four conics corresponding to $\Theta_{\beta, 1}$ for $\beta=0, \infty, \alpha_{1}, \alpha_{2}$, and eight lines corresponding to $\Theta_{\beta, 0}$ for $\beta=0, \infty, \alpha_{1}, \alpha_{2}, O, \Theta_{1,1}$, $\Theta_{1,2}$ and $\Theta_{1,3}$.

In the case $(\mathrm{g})$, there are eight reducible singular fibers of type III over the seven roots of the equation $\Delta(t)=0$ and $t=\infty$. Since the torsion-rank $r$ is 4 in this case, there are sixteen sections whose configuration together with an example of a single reducible singular fiber (the fiber over $t=0$ ), say, is shown in Figure 11.

In Figure 11, the seven sections $A_{1}, A_{2}, \ldots, A_{7}$ are those which intersect the same component of the fiber as the zero section. $T$ is a unique section intersecting $O$. Here, each section intersects only one other section. Hence one can divide sixteen sections 


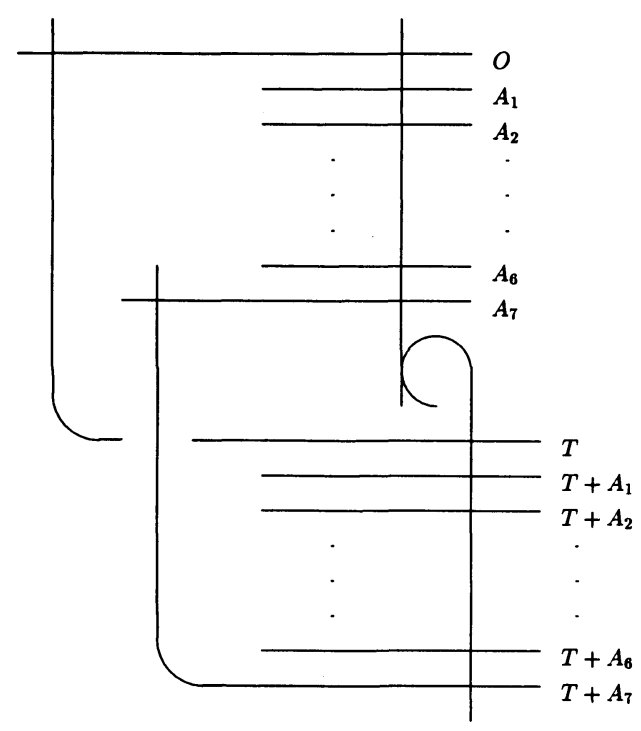

FIGURE 11.

into eight pairs, each consisting of two mutually intersecting sections.

Concretely, for the equation given in Table 1, we can put

$$
\begin{aligned}
T & =\left(\frac{t^{2}}{t^{2}+b}, \frac{a^{1 / 2} t^{4}+t^{3}+a^{1 / 2} b t^{2}}{\left(t+b^{1 / 2}\right)^{3}}\right) \\
A_{i} & =\left(u_{i}^{-1} t^{2}+u_{i} t+b u_{i}^{-1}, u_{i}^{-3 / 2}\left(t^{3}+\left(b+a u_{i}^{2}\right)^{1 / 2} t^{2}+b^{3 / 2}\right)\right) \quad(i=1,2,3) \\
A_{j+3} & =\left(v_{j} t+b v_{j}^{-1}, v_{j}^{-3 / 2}\left(v_{j}^{2} t^{2}+a^{1 / 2} b^{1 / 2} v_{j} t+b^{3 / 2}\right)\right) \quad(j=1, \ldots, 4),
\end{aligned}
$$

where $u_{i}(i=1,2,3)$ are the three roots of the equation $u^{3}+a u+1=0$ and $v_{j}(j=1, \ldots, 4)$ are the four roots of the equation $v^{4}+a v^{2}+v+b=0$. The same situation occurs on the other reducible singular fibers. If one names the fiber components in Figure 11 as in Figure 10 (the fiber on the left hand side), one can blow down the following nine sections and components so that one obtains a configuration on $\boldsymbol{P}^{2}$ consisting of $O, A_{1}$, $A_{2}, \ldots, A_{6}, T+A_{7}, \Theta_{0,1}$ in this order.

REMARK 5. The sections are all disjoint except in the case (g).

This is an immediate consequence of the formula in Proposition 4.5.

6. Calculation of the Mordell-Weil group. We introduce a method of calculating the Mordell-Weil groups in characteristic 2. We start with an equation

(6.1) $y^{2}=x^{3}+\varphi(t) x+\psi(t)$ with $\varphi(t), \psi(t) \in k[t]$ and either $\varphi(t) \notin K^{2}$ or $\psi(t) \notin K^{2}$ which satisfies 


$$
\min \left\{v_{\alpha}(\varphi(t))-4, v_{\alpha}(\psi(t))-6\right\}<0
$$

for any root $\alpha$ of $\Delta(t)=0$, where $v_{\alpha}$ is the $(t-\alpha)$-adic valuation with $v_{\alpha}(t-\alpha)=1$. Suppose $f(t), g(t)$ and $h(t)$ are polynomials in $k[t]$ such that $(f(t) / h(t), g(t) / h(t))$ is a solution of (6.1) and $\operatorname{gcd}(f(t), g(t), h(t))=1$. Substituting it into (6.1), we obtain

$$
g^{2} h=f^{3}+\varphi f h^{2}+\psi h^{3} .
$$

Here and in what follows $f, g, h, \varphi, \psi$, etc. stand for $f(t), g(t), h(t), \varphi(t), \psi(t)$ etc., for the sake of simplicity of the notation. First, we have

$$
h=h_{1}^{3}, \quad f=f_{1} h_{1} \quad \text { with } h_{1}, f_{1} \in k[t] \text { and } \operatorname{gcd}\left(f_{1}, h_{1}\right)=1
$$

in the same way as in characteristic 3 (cf. $[5, \S 5])$. Hence we have

$$
g^{2}=f_{1}^{3}+\varphi f_{1} h_{1}^{4}+\psi h_{1}^{6} \text {. }
$$

In view of (6.2) and Theorem 1.1, one gets

$$
\operatorname{deg} \varphi(t)<4 \chi, \quad \operatorname{deg} \psi(t)<6 \chi,
$$

where $\chi=\chi\left(\mathcal{O}_{X}\right)$ for a quasi-elliptic surface $X$ defined by the equation (6.1). Now, let us consider an equation in the form

$$
G(t)^{2}=F(t)^{3}+\Phi(t) F(t)+\Psi(t),
$$

where $F(t), G(t), \Phi(t), \Psi(t) \in k[t], \Phi(t) \notin k[t]^{2}$ or $\Psi(t) \notin k[t]^{2}$.

Then we have the following:

LEMMA 6.1. (i) If $F^{\prime} \neq 0$ then

$$
\begin{aligned}
& \operatorname{deg} F \leqslant \max \left\{\operatorname{deg} \Phi-1, \frac{1}{2} \operatorname{deg} \Psi-\frac{1}{2}\right\} \\
& \operatorname{deg} G \leqslant \frac{1}{2} \max \{3 \operatorname{deg} F, \operatorname{deg} \Phi+\operatorname{deg} F, \operatorname{deg} \Psi\} .
\end{aligned}
$$

(ii) If $F^{\prime}=0$ then,

$$
\begin{aligned}
& F=G=0 \quad \text { when } \quad \Psi^{\prime}=0, \\
& \operatorname{deg} F \leqslant \operatorname{deg} \Psi-1 \text { when } \Psi^{\prime} \neq 0 .
\end{aligned}
$$

Proof. Differentiating both sides of (6.5), we have

$$
F^{2} F^{\prime}+(\Phi F)^{\prime}+\Psi^{\prime}=0 \text {. }
$$

Thus $F^{2}$ divides $(\Phi F)^{\prime}+\Psi^{\prime}$, which implies

$2 \operatorname{deg} F \leqslant \max \{\operatorname{deg} \Phi+\operatorname{deg} F-1, \operatorname{deg} \Psi-1\}$, whenever $\quad F^{\prime} \neq 0$. 
So we obtain (i). If $F^{\prime}=0,(6.6)$ becomes

$$
\Phi^{\prime} \cdot F+\Psi^{\prime}=0 \text {. }
$$

We are done by the assumptions on $\Phi$ and $\Psi$.

Now applying this lemma to $F=f_{1}, G=g, \Phi=\varphi h^{4}, \Psi=\psi h_{1}^{6}$, and making use of (6.4), we have

$$
\operatorname{deg} f_{1} \leqslant 4(\chi+d)-2, \quad \operatorname{deg} g \leqslant 6(\chi+d)-3,
$$

where $d=\operatorname{deg} h_{1}$. Note that a bound for $d$ can be obtained by looking at the configuration of reducible singular fibers. More precisely, we have the following:

LEMMA 6.2. Let $P(\neq O)$ be a rational point of the form $\left(f_{1}(t) / h_{1}(t)^{2}, g(t) / h_{1}(t)^{3}\right)$ on an affine open set and let $d=\operatorname{deg} h_{1}$. Then the following inequality holds:

$$
d \leqslant \frac{1}{4} v(\mathrm{III})+\frac{3}{4} v\left(\mathrm{III}^{*}\right)+\sum_{b \geqslant 0}\left(\frac{1}{2}+\frac{b}{8}\right) v\left(\mathrm{I}_{b}^{*}\right)-\chi .
$$

Proof. Suppose $(P)$ and $(O)$ intersect each other on the fiber over $v \in \boldsymbol{P}^{1}$. Then the $x$-coordinate $f_{1}(t) / h_{1}(t)^{2}$ of $P$ must have a pole at $t=v$ if $v \neq \infty$, since the section $(O)$ is taken as the section at infinity of the affine open set. Let $v$ be a root of $h_{1}(t)=0$. Then, since $\left(f_{1}(t), h_{1}(t)\right)=1$, the $x$-coordinate $f_{1}(t) / h_{1}(t)^{2}$ of $P$ has a pole of order equal to the multiplicity of a root $v$ in $h_{1}(t)^{2}=0$, and $(P)$ intersects $(O)$ on the fiber over $t=v$ with multiplicity equal to the multiplicity of the root $v$ in $h_{1}(t)=0$. Thus we have the inequality $d \leqslant(P \cdot O)$. The inequality in the statement follows from this with the formula in Proposition 4.5 taken into account.

\section{REFERENCES}

[1] M. Artin, On Enriques' surfaces, thesis, Harvard University, (1960).

[2] M. Artin, Supersingular K3 surfaces, Ann. scient. École Norm. Sup. $4^{\text {e }}$ Ser. 7 (1974), 543-568.

[ 3 ] E. BOMBIERI AND D. HUSEMOLLER, Classification and embeddings of surfaces, Proceedings of Symposia in Pure Mathematics 29 (1975), Amer. Math. Soc., 329-420.

[4] F. R. Cossec AND I. V. Dolgachev, Enriques Surfaces I, Progress in Math. 76, Birkhäuser, Boston-Basel-Berlin (1989).

[ 5] H. Iто, The Mordell-Weil groups of unirational quasi-elliptic surfaces in characteristic 3, Math. Z. 211 (1992), 1-40.

[6] K. KodAirA, On compact analytic surfaces II-III, Ann. of Math. 77 (1963), 563-626; ibid. 78 (1963), $1-40$.

[ 7 ] M. MiYanishi, Unirational quasi-elliptic surfaces, Japan. J. Math. 3 (1977), 395- 416.

[8] T. ShIOdA, Mordell-Weil lattices and Galois representation I, II, III, Proc. Japan Acad. 65A (1989), 268-271; 296-299; 300-303.

[9] T. Shioda, On the Mordell-Weil lattices, Comment. Math. Univ. St. Pauli 39 (1990), 211-240.

[10] J. TATE, Genus change in inseparable extensions of function fields, Proc. Amer. Math. Soc. 3 (1952), $400-406$. 
Mathematical Institute

FACUlTy OF SCIENCE

TÔHOKU UNIVERSITY

SENDAI 980-77

JAPAN

E-mail address: hiroito@math.tohoku.ac.jp 
